\title{
Multiple-scattering calculations for layered phononic structures of nonspherical particles
}

\author{
G. Gantzounis and N. Papanikolaou \\ Institute of Microelectronics, NCSR “Demokritos,” GR-153 10 Athens, Greece
}

\author{
N. Stefanou \\ University of Athens, Section of Solid State Physics, Panepistimioupolis, GR-157 84 Athens, Greece
}

(Received 28 December 2010; published 6 June 2011)

\begin{abstract}
We present an extension of the layer-multiple-scattering method to phononic crystals of nonspherical particles in a homogeneous host medium by employing the extended-boundary-condition technique for the description of the individual scatterers. The efficiency of the method is demonstrated on specific examples of two- and three-dimensional periodic assemblies of spheroidal polymer particles in water and in silicon. We report a thorough analysis of the acoustic properties of these composites and emphasize aspects of the underlying physics that relate to the nonspherical shape of the particles.
\end{abstract}

DOI: 10.1103/PhysRevB.83.214301

PACS number(s): 46.40.Cd, 43.40.+s, 43.20.+g

\section{INTRODUCTION}

In composite materials with periodic modulation in their elastic properties, so-called phononic crystals, the propagation of acoustic waves can be distinctly different from wave propagation in their isotropic bulk constituents, leading to intriguing phenomena and exciting applications. ${ }^{1}$ One of the most interesting properties of phononic crystals is the existence of frequency band gaps when the sound wavelengths commensurate the structure periodicity. Audible sound is modified by meter-sized structures, ${ }^{2}$ while submicron lattices are active for hypersound ( $\mathrm{GHz}$ frequencies). ${ }^{3}$

The layer-multiple-scattering (LMS) method is a very powerful computational methodology for studying threedimensional (3D) phononic crystals consisting of nonoverlapping spherical particles (scatterers) in a homogeneous host medium $^{4-6}$ and applies to any material type combination (fluid or solid). An analogous method for two-dimensional (2D) phononic crystals of infinite cylinders has also been reported. ${ }^{7}$ An important aspect of the method is that, contrary to traditional band-structure ${ }^{8-11}$ or time-domain ${ }^{12-14}$ methods, it solves the wave equation of elasticity in frequency domain and thus one can allow the elastic coefficients of any of the constituent materials to depend on the frequency, including also absorptive losses. ${ }^{15-18}$ Besides the complex phononic band structure of an infinite crystal, associated with a given crystallographic plane, the LMS method can also provide the transmission, reflection, and absorption coefficients of an elastic wave of any polarization incident at a given angle on a finite slab of the crystal and, therefore, it can describe an actual transmission experiment. A further advantage of the method is that it does not require periodicity in the direction perpendicular to the layers, which must only have the same 2D periodicity. Therefore, a number of interesting configurations, such as planar defects, ${ }^{19}$ heterostructures, ${ }^{6}$ stacking disorder, ${ }^{20}$ phononic crystal slabs on homogeneous plates, and semi-infinite substrates, ${ }^{21,22}$ can be treated in a more or less straightforward manner. Finally, the LMS method can incorporate multiple-scattering Green's-function techniques ${ }^{23}$ that allow one to calculate the (local) density of states of the elastic field, as well as to treat defects, disorder, and the system response to an external perturbation in general.
The LMS method has been proven very efficient for phononic crystals of spherical particles. ${ }^{15-22,24-28}$ With the same efficiency, it can treat, also, coated spheres ${ }^{29-32}$ and generally spherical particles consisting of an arbitrary number of concentric spherical shells by a powerful recursive algorithm. ${ }^{6}$ On the other hand, nonspherical building units offer more freedom for engineering the elastic field eigenmodes and, nowadays, such structures can be fabricated also at the nanoscale using modern nanofabrication techniques. ${ }^{33-38}$ In these cases, other computational methodologies, such as the plane-wave-expansion method m $^{8,149}$ or finite-difference techniques, ${ }^{40-42}$ are applicable. However, these methods do not easily allow the gain of physical insight while in many situations they are computationally cumbersome. In addition to physical clarity and computational efficiency, the generalized LMS method retains all the advantages listed in the previous paragraph versus more traditional methods, such as the plane-wave-expansion and the finite-difference-timedomain methods. Therefore, extension of the LMS method to scatterers of arbitrary shape would offer an efficient and versatile alternative to treat a variety of complex phononic structures. Such an extension does not involve major difficulties, since the scattering properties of the individual particles enter only through the corresponding $T$ matrix, which can be efficiently evaluated by the extended-boundary-condition (EBC) technique ${ }^{43-45}$ for particles of arbitrary shape, at least for not-too-large deviations from the spherical shape, such as spheroids, capped cylinders, etc. ${ }^{46-48}$ Recently, Ivansson incorporated successfully Boström's EBC algorithm ${ }^{45}$ into the LMS method to calculate the reflectance of a monolayer of nonspherical voids in a rubber material and discussed the convergence and the efficiency of the implementation. ${ }^{49,50}$

In this paper, we report a generalization of the full LMS methodology to phononic structures of nonspherical particles in a homogeneous host medium, for material combinations of any type, fluid or solid, including also the limiting cases of the void and the perfectly rigid body. We employ the EBC technique for the evaluation of the $T$ matrix of the individual scatterers ${ }^{43,45}$ properly adapted to our fomalism. In this respect, we note that a corresponding extended LMS method for photonic crystals is already available, ${ }^{51}$ and the ability to 
treat on equal footing the electromagnetic and the elastic fields by the same methodological approach in appropriately designed structures, so-called phoxonic crystals, ${ }^{39,52}$ offers unique advantages. This is an additional motivation for the present work. The remainder of the paper is organized as follows. Section II summarizes the basic concepts and formulas of elastodynamics of a homogeneous medium. In Sec. III we present the EBC formalism adapted to our LMS method and report in the appendix equations specific for the evaluation of the scattering $T$ matrix for a fluid, solid, perfectly rigid, and void scatterer in a fluid or solid matrix. Applications on a spheroidal polymer particle in a fluid and a solid environment are discussed in comparison to a corresponding spherical particle. Section IV is devoted to the description of elastic wave scattering from finite slabs of periodically arranged nonspherical particles, including the analysis of the elastodynamic response of a 2D periodic assembly of spheroidal polymer particles in a fluid and a solid environment by means of relevant transmission spectra and density of states diagrams. Section V deals with the complex phononic band structure of corresponding infinite crystals. We discuss the physical origin of the elastic field eigenmodes in conjunction with corresponding transmission spectra of finite slabs of these crystals, in the specific examples under consideration, with the help of group theory and provide a consistent interpretation of the underlying physics. The main results of the article are summarized in the last section.

\section{ELASTIC WAVES IN A HOMOGENEOUS MEDIUM}

The displacement vector field, $\mathbf{U}(\mathbf{r}, t)$, in a homogeneous elastic medium characterized by constant mass density $\rho$ and Lamé coefficients $\lambda, \mu$, satisfies the equation of motion ${ }^{53}$

$$
\begin{aligned}
\rho \partial_{t}^{2} U_{i}(\mathbf{r}, t)= & \sum_{i^{\prime \prime}} \partial_{i^{\prime \prime}}\left\{\lambda \delta_{i i^{\prime \prime}} \sum_{i^{\prime}} \partial_{i^{\prime}} U_{i^{\prime}}(\mathbf{r}, t)\right. \\
& \left.+\mu\left[\partial_{i^{\prime \prime}} U_{i}(\mathbf{r}, t)+\partial_{i} U_{i^{\prime \prime}}(\mathbf{r}, t)\right]\right\},
\end{aligned}
$$

where $\partial_{i}$ denotes partial derivative with respect to the $i$ th component of the position vector $\mathbf{r}$ and $\partial_{t}$ denotes partial derivative with respect to time. For a harmonic elastic wave of angular frequency $\omega, \mathbf{U}(\mathbf{r}, t)=\operatorname{Re}[\mathbf{u}(\mathbf{r}) \exp (-\mathrm{i} \omega t)]$, Eq. (1) reduces to the time-independent form,

$$
\sum_{i^{\prime \prime}} \partial_{i^{\prime \prime}} \sigma_{i i^{\prime \prime}}(\mathbf{r})+\rho \omega^{2} u_{i}(\mathbf{r})=0,
$$

where

$$
\sigma_{i i^{\prime \prime}}(\mathbf{r})=\lambda \delta_{i i^{\prime \prime}} \sum_{i^{\prime}} \partial_{i^{\prime}} u_{i^{\prime}}(\mathbf{r})+\mu\left[\partial_{i^{\prime \prime}} u_{i}(\mathbf{r})+\partial_{i} u_{i^{\prime \prime}}(\mathbf{r})\right]
$$

is the stress tensor. Since, as can be seen from Eq. (3), $\sigma_{i i^{\prime \prime}}(\mathbf{r})=$ $\sigma_{i^{\prime \prime} i}(\mathbf{r})$, Eq. (2) can be written in the more compact form,

$$
\nabla \cdot \boldsymbol{\sigma}(\mathbf{r})+\rho \omega^{2} \mathbf{u}(\mathbf{r})=0
$$

with the $i$ th component of the vector $\nabla \cdot \sigma(\mathbf{r})$ defined in the usual manner: $[\nabla \cdot \sigma(\mathbf{r})]_{i}=\sum_{i^{\prime \prime}} \partial_{i^{\prime \prime}} \sigma_{i^{\prime \prime} i}(\mathbf{r})$. On the other hand, the surface traction, $\mathbf{t}(\mathbf{r})$, on a surface $S$, with a normal unit vector $\hat{\mathbf{n}}$, is given by $\mathbf{t}(\mathbf{r})=\hat{\mathbf{n}} \cdot \boldsymbol{\sigma}(\mathbf{r})$.
The most general solution of Eq. (4) consists of two kinds of elastic waves that propagate independently: (a) longitudinal (irrotational) waves, which satisfy the equations

$$
\left(\nabla^{2}+q_{l}^{2}\right) \mathbf{u}(\mathbf{r})=0, \quad \nabla \times \mathbf{u}=0,
$$

where $q_{l}=\omega / c_{l}, \quad c_{l}=\sqrt{(\lambda+2 \mu) / \rho}$ being the speed of propagation of this wave; and (b) transverse (divergenceless) waves, which satisfy the equations

$$
\left(\nabla^{2}+q_{t}^{2}\right) \mathbf{u}(\mathbf{r})=0, \quad \nabla \cdot \mathbf{u}=0,
$$

where $q_{t}=\omega / c_{t}, c_{t}=\sqrt{\mu / \rho}$ being the speed of propagation of this wave.

One can define a complete set of plane-wave solutions of Eqs. (5) and (6) as follows:

$$
\mathbf{u}_{\mathbf{q p}}(\mathbf{r})=u_{0} \hat{\mathbf{e}}_{\mathrm{p}}\left(\mathbf{q}_{v}\right) \exp \left(\mathbf{i q} \mathbf{q}_{v} \mathbf{r}\right),
$$

where $v=l(t)$ for longitudinal (transverse) waves, $u_{0}$ is the magnitude and $\hat{\mathbf{e}}_{\mathrm{p}}\left(\mathbf{q}_{v}\right)$ is the polarization unit vector of the elastic field. The subscript $\mathrm{p}$ takes the values $\mathrm{p}=1,2,3 ; \mathrm{p}=1$ is the radial unit vector along $\mathbf{q}_{l}$ and applies to longitudinal waves, and $p=2$ and $p=3$ are the polar and azimuthal unit vectors, respectively, which are perpendicular to $\mathbf{q}_{t}$ and refer to transverse waves.

Alternatively, one can define a complete set of sphericalwave solutions of Eq. (5) and (6). Irrotational vector wave functions, given by ${ }^{23}$

$$
\begin{aligned}
\mathbf{J}_{0 \ell m}(\mathbf{r}) & =\frac{1}{q_{l}} \nabla\left[j_{\ell}\left(q_{l} r\right) Y_{\ell m}(\hat{\mathbf{r}})\right], \\
\mathbf{H}_{0 \ell m}(\mathbf{r}) & =\frac{1}{q_{l}} \nabla\left[h_{\ell}^{+}\left(q_{l} r\right) Y_{\ell m}(\hat{\mathbf{r}})\right],
\end{aligned}
$$

where $j_{\ell}$ and $h_{\ell}^{+}$are the spherical Bessel and Hankel functions, respectively, and $Y_{\ell m}(\hat{\mathbf{r}})$ are the usual spherical harmonics with $\hat{\mathbf{r}}$ denoting the angular variables $(\theta, \phi)$ of $\mathbf{r}$ in a system of spherical coordinates, satisfy Eq. (5). Correspondingly, solenoidal vector wave functions, given by ${ }^{23}$

$$
\mathbf{J}_{1 \ell m}(\mathbf{r})=j_{\ell}\left(q_{t} r\right) \mathbf{X}_{\ell m}(\hat{\mathbf{r}}), \quad \mathbf{H}_{1 \ell m}(\mathbf{r})=h_{\ell}^{+}\left(q_{t} r\right) \mathbf{X}_{\ell m}(\hat{\mathbf{r}}),
$$

and

$$
\begin{aligned}
\mathbf{J}_{2 \ell m}(\mathbf{r}) & =\frac{\mathrm{i}}{q_{t}} \nabla \times \mathbf{J}_{1 \ell m}(\mathbf{r}), \\
\mathbf{H}_{2 \ell m}(\mathbf{r}) & =\frac{\mathrm{i}}{q_{t}} \nabla \times \mathbf{H}_{1 \ell m}(\mathbf{r}),
\end{aligned}
$$

are appropriate solutions of Eq. (6). The so-called vector spherical harmonics $\mathbf{X}_{\ell m}(\hat{\mathbf{r}})$, which appear in Eqs. (9) and (10), are defined by $\sqrt{\ell(\ell+1)} \mathbf{X}_{\ell m}(\hat{\mathbf{r}})=-\mathrm{i} \hat{\mathbf{r}} \times \nabla Y_{\ell m}(\hat{\mathbf{r}})$. Explicit expressions for the vector spherical harmonics are given elsewhere. $^{6}$

At a given frequency, the most general form of displacement field can be written as a linear combination of the regular, $\mathbf{J}_{\mathrm{L}}(\mathbf{r})$, and the irregular at the origin $(r=0), \mathbf{H}_{\mathrm{L}}(\mathbf{r})$, vector spherical waves, that is,

$$
\mathbf{u}(\mathbf{r})=\sum_{\mathrm{L}}\left[a_{\mathrm{L}}^{0} \mathbf{J}_{\mathrm{L}}(\mathbf{r})+a_{\mathrm{L}}^{+} \mathbf{H}_{\mathrm{L}}(\mathbf{r})\right]
$$

where $\mathrm{L}$ denotes collectively the indices $P \ell m . P=0,1,2$ is the polarization of the spherical wave and should not be 
confused with $\mathrm{p}$, which denotes the polarization of a plane wave. For example, a longitudinal plane wave of wave vector $\mathbf{q}_{l}$, defined by Eq. (7), is finite everywhere. Therefore, it can be expanded into irrotational regular spherical waves, $\mathbf{J}_{0 \ell m}(\mathbf{r})$, with coefficients

$$
a_{0 \ell m}^{0}=u_{0} \mathbf{A}_{0 \ell m}^{0}\left(\hat{\mathbf{q}}_{l}\right) \cdot \hat{\mathbf{e}}_{1}\left(\mathbf{q}_{l}\right),
$$

where $\mathbf{A}_{0 \ell m}^{0}\left(\hat{\mathbf{q}}_{l}\right)=4 \pi \mathrm{i}^{\ell+1}(-1)^{m+1} Y_{\ell-m}\left(\hat{\mathbf{q}}_{l}\right) \hat{\mathbf{e}}_{1}\left(\mathbf{q}_{l}\right)$. Similarly, a transverse plane wave of wave vector $\mathbf{q}_{t}$ can be expanded into solenoidal regular spherical waves, $\mathbf{J}_{1 \ell m}(\mathbf{r})$ and $\mathbf{J}_{2 \ell m}(\mathbf{r})$, with coefficients

$$
a_{P \ell m}^{0}=u_{0} \mathbf{A}_{P \ell m}^{0}\left(\hat{\mathbf{q}}_{t}\right) \cdot \hat{\mathbf{e}}_{\mathrm{p}}\left(\mathbf{q}_{t}\right), \quad P=1,2,
$$

where $\mathbf{A}_{1 \ell m}^{0}\left(\hat{\mathbf{q}}_{t}\right)=4 \pi \mathrm{i}^{\ell}(-1)^{m+1} \mathbf{X}_{\ell-m}\left(\hat{\mathbf{q}}_{t}\right)$ and $\mathbf{A}_{2 \ell m}^{0}\left(\hat{\mathbf{q}}_{t}\right)=$ $4 \pi \mathrm{i}^{\ell}(-1)^{m+1} \mathbf{X}_{\ell-m}\left(\hat{\mathbf{q}}_{t}\right) \times \hat{\mathbf{e}}_{1}\left(\mathbf{q}_{t}\right)$.

Equation (4) can be cast into the form of an eigenvalue problem,

$$
\boldsymbol{\Lambda}(\mathbf{r}) \mathbf{u}(\mathbf{r})=\omega^{2} \mathbf{u}(\mathbf{r}),
$$

of the tensor differential operator $\boldsymbol{\Lambda}(\mathbf{r})$, defined by

$$
\Lambda_{i i^{\prime}}(\mathbf{r})=-\frac{1}{\rho}\left[\lambda \partial_{i} \partial_{i^{\prime}}+\mu\left(\delta_{i i^{\prime}} \sum_{i^{\prime \prime}} \partial_{i^{\prime \prime}}^{2}+\partial_{i^{\prime}} \partial_{i}\right)\right],
$$

which operates on the Hilbert space of square integrable vector functions. The associated retarded Green's dyadic, $\mathbf{G}\left(\mathbf{r}, \mathbf{r}^{\prime}\right)$, is defined by

$$
\sum_{i}\left[\omega^{2} \delta_{i^{\prime \prime} i}-\Lambda_{i^{\prime \prime} i}(\mathbf{r})\right] G_{i i^{\prime}}\left(\mathbf{r}, \mathbf{r}^{\prime}\right)=\delta_{i^{\prime \prime} i^{\prime}} \delta\left(\mathbf{r}-\mathbf{r}^{\prime}\right)
$$

where $\omega^{2} \equiv \lim _{\epsilon \rightarrow 0^{+}}(\omega+\mathrm{i} \epsilon)^{2}$, and can be expressed into vector spherical waves as follows: ${ }^{23}$

$$
\begin{aligned}
G_{i i^{\prime}}\left(\mathbf{r}, \mathbf{r}^{\prime}\right)= & -\mathrm{i} \sum_{\mathrm{L}} \frac{\omega}{c_{v}^{3}}\left[\bar{J}_{\mathrm{L} ; i}(\mathbf{r}) H_{\mathrm{L} ; i^{\prime}}(\mathbf{r}) \Theta\left(r^{\prime}-r\right)\right. \\
& \left.+\bar{H}_{\mathrm{L} ; i}(\mathbf{r}) J_{\mathrm{L} ; i^{\prime}}(\mathbf{r}) \Theta\left(r-r^{\prime}\right)\right],
\end{aligned}
$$

where $v=l$ if $P=0$ and $v=t$ if $P=1,2 . \Theta(x)$ is the Heaviside step function and $\overline{\mathbf{F}}_{P \ell m}(\mathbf{r})=(-1)^{P+m} \mathbf{F}_{P \ell-m}(\mathbf{r})$, with $\mathbf{F}_{P \ell m}$ denoting $\mathbf{J}_{P \ell m}$ or $\mathbf{H}_{P \ell m}$. It is worth noting that $G_{i i^{\prime}}\left(\mathbf{r}, \mathbf{r}^{\prime}\right)=G_{i^{\prime} i}\left(\mathbf{r}^{\prime}, \mathbf{r}\right)$. We further define the Green's stress triadic, $\boldsymbol{\Sigma}\left(\mathbf{r}, \mathbf{r}^{\prime}\right)$, in a manner similar to Eq. (3), as follows:

$$
\begin{aligned}
\Sigma_{i i^{\prime} i^{\prime \prime}}\left(\mathbf{r}, \mathbf{r}^{\prime}\right)= & \lambda \delta_{i i^{\prime}} \sum_{i^{\prime \prime \prime}} \partial_{i^{\prime \prime \prime}} G_{i^{\prime \prime \prime} i^{\prime \prime}}\left(\mathbf{r}, \mathbf{r}^{\prime}\right) \\
& +\mu\left[\partial_{i^{\prime}} G_{i i^{\prime \prime}}\left(\mathbf{r}, \mathbf{r}^{\prime}\right)+\partial_{i} G_{i^{\prime} i^{\prime \prime}}\left(\mathbf{r}, \mathbf{r}^{\prime}\right)\right] .
\end{aligned}
$$

It is straightforward to show that the Green's stress triadic is symmetric, that is, $\Sigma_{i i^{\prime} i^{\prime \prime}}\left(\mathbf{r}, \mathbf{r}^{\prime}\right)=\Sigma_{i^{\prime} i i^{\prime \prime}}\left(\mathbf{r}, \mathbf{r}^{\prime}\right)$, and $\mathbf{G}\left(\mathbf{r}, \mathbf{r}^{\prime}\right)$ is related to $\Sigma\left(\mathbf{r}, \mathbf{r}^{\prime}\right)$ through the equation

$$
\boldsymbol{\Lambda}(\mathbf{r}) \mathbf{G}\left(\mathbf{r}, \mathbf{r}^{\prime}\right)=-\frac{1}{\rho} \nabla \cdot \mathbf{\Sigma}\left(\mathbf{r}, \mathbf{r}^{\prime}\right) .
$$

Using Eqs. (4), (16), and (19), it can be shown that ${ }^{54}$

$$
\nabla \cdot\left[\boldsymbol{\sigma}(\mathbf{r}) \cdot \mathbf{G}\left(\mathbf{r}, \mathbf{r}^{\prime}\right)-\mathbf{u}(\mathbf{r}) \cdot \boldsymbol{\Sigma}\left(\mathbf{r}, \mathbf{r}^{\prime}\right)\right]=-\rho \mathbf{u}(\mathbf{r}) \delta\left(\mathbf{r}-\mathbf{r}^{\prime}\right) .
$$

Furthermore, from Eq. (18), with the help of Eq. (17), it is straightforward to show that

$$
\begin{aligned}
\hat{\mathbf{n}} \cdot \boldsymbol{\Sigma}\left(\mathbf{r}, \mathbf{r}^{\prime}\right)= & \lambda \hat{\mathbf{n}} \nabla \cdot \mathbf{G}\left(\mathbf{r}, \mathbf{r}^{\prime}\right) \\
& +2 \mu(\hat{\mathbf{n}} \cdot \nabla) \mathbf{G}\left(\mathbf{r}, \mathbf{r}^{\prime}\right)+\mu \hat{\mathbf{n}} \times\left[\nabla \times \mathbf{G}\left(\mathbf{r}, \mathbf{r}^{\prime}\right)\right] \\
= & -\mathrm{i} \sum_{\mathrm{L}} \frac{\omega}{c_{v}^{3}}\left\{\mathbf{t}\left[\overline{\mathbf{J}}_{\mathrm{L}}(\mathbf{r})\right] \mathbf{H}_{\mathrm{L}}\left(\mathbf{r}^{\prime}\right) \Theta\left(r^{\prime}-r\right)\right. \\
& \left.+\mathbf{t}\left[\overline{\mathbf{H}}_{\mathrm{L}}(\mathbf{r})\right] \mathbf{J}_{\mathrm{L}}\left(\mathbf{r}^{\prime}\right) \Theta\left(r-r^{\prime}\right)\right\}
\end{aligned}
$$

with the surface traction associated with a vector spherical wave $\mathbf{F}_{\mathrm{L}}(\mathbf{r})$ given by ${ }^{45}$

$$
\begin{aligned}
\mathbf{t}\left[\mathbf{F}_{\mathrm{L}}(\mathbf{r})\right]= & \lambda \hat{\mathbf{n}} \nabla \cdot \mathbf{F}_{\mathrm{L}}(\mathbf{r})+2 \mu(\hat{\mathbf{n}} \cdot \nabla) \mathbf{F}_{\mathrm{L}}(\mathbf{r}) \\
& +\mu \hat{\mathbf{n}}\left[\nabla \times \mathbf{F}_{\mathrm{L}}(\mathbf{r})\right] .
\end{aligned}
$$

\section{SINGLE-PARTICLE SCATTERING}

We assume a nonspherical, in general, scatterer made of a homogeneous material with mass density $\rho_{\mathrm{s}}$ and Lamé coefficients $\lambda_{\mathrm{s}}, \mu_{\mathrm{s}}$. The scatterer is embedded in an infinite homogeneous medium characterized by elastic parameters $\rho$, $\lambda, \mu$, and occupies a volume $V_{\mathrm{s}}$, with a boundary surface $S$ defined by a normal unit vector $\hat{\mathbf{n}}$ pointing outward. Integrating Eq. (20) outside the volume $V_{\mathrm{s}}$ and applying Green's theorem we obtain

$\frac{1}{\rho} \oint_{S} d S\left\{\mathbf{t}_{+}(\mathbf{r}) \cdot \mathbf{G}\left(\mathbf{r}, \mathbf{r}^{\prime}\right)-\mathbf{u}_{+}(\mathbf{r}) \cdot\left[\hat{\mathbf{n}} \cdot \boldsymbol{\Sigma}\left(\mathbf{r}, \mathbf{r}^{\prime}\right)\right]\right\}-\frac{1}{\rho} \oint_{S_{\infty}} d S\left\{\mathbf{t}_{1}(\mathbf{r}) \cdot \mathbf{G}\left(\mathbf{r}, \mathbf{r}^{\prime}\right)-\mathbf{u}_{1}(\mathbf{r}) \cdot\left[\hat{\mathbf{n}}_{1} \cdot \boldsymbol{\Sigma}\left(\mathbf{r}, \mathbf{r}^{\prime}\right)\right]\right\}= \begin{cases}\mathbf{u}\left(\mathbf{r}^{\prime}\right) & \text { if } \mathbf{r}^{\prime} \text { outside } V_{\mathrm{s}}, \\ 0 & \text { if } \mathbf{r}^{\prime} \text { inside } V_{\mathrm{s}},\end{cases}$

where $\mathbf{u}_{+}(\mathbf{r})$ and $\mathbf{t}_{+}(\mathbf{r})$ are the limiting values of the displacement field and the surface traction, respectively, on the surface $S$, taken from outside. Similarly, the displacement field, $\mathbf{u}_{1}(\mathbf{r})$, and the surface traction, $\mathbf{t}_{1}(\mathbf{r})$, are calculated on a surface $S_{\infty}$ surrounding the particle at an infinite distance, defined by the normal unit vector $\hat{\mathbf{n}}_{1}$ pointing outward. In Eq. (23) we made use of the symmetric form of $\boldsymbol{\Sigma}$, which implies that $\mathbf{u}(\mathbf{r}) \cdot\left[\hat{\mathbf{n}} \cdot \boldsymbol{\Sigma}\left(\mathbf{r}, \mathbf{r}^{\prime}\right)\right]=\hat{\mathbf{n}} \cdot\left[\mathbf{u}(\mathbf{r}) \cdot \boldsymbol{\Sigma}\left(\mathbf{r}, \mathbf{r}^{\prime}\right)\right]$.

On the other hand, integrating Eq. (20) inside the volume $V_{\mathrm{s}}$, and applying Green's theorem we obtain

$$
-\frac{1}{\rho_{\mathrm{s}}} \oint_{S} d S\left\{\mathbf{t}_{-}(\mathbf{r}) \cdot \mathbf{G}^{\mathrm{s}}\left(\mathbf{r}, \mathbf{r}^{\prime}\right)-\mathbf{u}_{-}(\mathbf{r}) \cdot\left[\hat{\mathbf{n}} \cdot \boldsymbol{\Sigma}^{\mathrm{s}}\left(\mathbf{r}, \mathbf{r}^{\prime}\right)\right]\right\}= \begin{cases}\mathbf{u}\left(\mathbf{r}^{\prime}\right) & \text { if } \mathbf{r}^{\prime} \text { inside } V_{\mathrm{s}}, \\ 0 & \text { if } \mathbf{r}^{\prime} \text { outside } V_{\mathrm{s}},\end{cases}
$$


where $\mathbf{u}_{-}(\mathbf{r})$ and $\mathbf{t}_{-}(\mathbf{r})$ are the limiting values of the displacement field and the surface traction, respectively, on the surface $S$, taken from inside, and the index s denotes the medium of the scatterer. From Eq. (24) it is straightforward to show that the interior surface fields satisfy the integral equation

$$
\oint_{S} d S\left\{\mathbf{u}_{-}(\mathbf{r}) \cdot \mathbf{t}\left[\overline{\mathbf{J}}_{\mathrm{L}}^{\mathrm{s}}(\mathbf{r})\right]-\mathbf{t}_{-}(\mathbf{r}) \cdot \overline{\mathbf{J}}_{\mathrm{L}}^{\mathrm{s}}(\mathbf{r})\right\}=0 .
$$

We next consider a plane wave, $\mathbf{u}_{0}(\mathbf{r})$, incident on the scatterer. This triggers a scattered field, $\mathbf{u}_{\mathrm{sc}}(\mathbf{r})$. In general, the incident and scattered fields, which constitute the total field outside the scatterer, can be expanded into vector spherical waves as follows ${ }^{4}$ :

$$
\mathbf{u}_{0}(\mathbf{r})=\sum_{\mathrm{L}} a_{\mathrm{L}}^{0} \mathbf{J}_{\mathrm{L}}(\mathbf{r}), \quad \mathbf{u}_{\mathrm{sc}}(\mathbf{r})=\sum_{\mathrm{L}} a_{\mathrm{L}}^{+} \mathbf{H}_{\mathrm{L}}(\mathbf{r}) .
$$

Since the scattered field vanishes at infinity, only the incident field enters into the integral over the surface $S_{\infty}$ in Eq. (23). On the other hand, since in the absence of the scatterer the first integral on the left-hand side of Eq. (23) vanishes, the integral over $S_{\infty}$ equals the incident field at $\mathbf{r}^{\prime}, \mathbf{u}_{0}\left(\mathbf{r}^{\prime}\right)$. Therefore, inserting Eqs. (17) and (21) into Eq. (23) we obtain

$$
\begin{aligned}
\mathbf{u}_{0}\left(\mathbf{r}^{\prime}\right)= & \mathrm{i} \sum_{\mathrm{L}} \frac{q_{v}}{\rho c_{v}^{2}} \oint_{S} d S\left\{\mathbf{t}_{+}(\mathbf{r}) \cdot \overline{\mathbf{H}}_{\mathrm{L}}(\mathbf{r})\right. \\
& \left.-\mathbf{u}_{+}(\mathbf{r}) \cdot \mathbf{t}\left[\overline{\mathbf{H}}_{\mathrm{L}}(\mathbf{r})\right]\right\} \mathbf{J}_{\mathrm{L}}\left(\mathbf{r}^{\prime}\right)
\end{aligned}
$$

for $\mathbf{r}^{\prime}$ inside the sphere inscribed into $V_{\mathrm{s}}$ and

$$
\begin{aligned}
\mathbf{u}_{\mathrm{sc}}\left(\mathbf{r}^{\prime}\right)= & -\mathrm{i} \sum_{\mathrm{L}} \frac{q_{v}}{\rho c_{v}^{2}} \oint_{S} d S\left\{\mathbf{t}_{+}(\mathbf{r}) \cdot \overline{\mathbf{J}}_{\mathrm{L}}(\mathbf{r})\right. \\
& \left.-\mathbf{u}_{+}(\mathbf{r}) \cdot \mathbf{t}\left[\overline{\mathbf{J}}_{\mathrm{L}}(\mathbf{r})\right]\right\} \mathbf{H}_{\mathrm{L}}\left(\mathbf{r}^{\prime}\right)
\end{aligned}
$$

for $\mathbf{r}^{\prime}$ outside the sphere circumscribing $V_{\mathrm{s}}$, where $v=l$ if $P=0$ and $v=t$ if $P=1,2$. Comparing Eqs. (26) with Eqs. (27) and (28) we identify the following expressions for the coefficients of the incident and the scattered waves,

$$
\begin{array}{r}
a_{\mathrm{L}}^{0}=\frac{-\mathrm{i} q_{v}}{\rho c_{v}^{2}} \oint_{S} d S\left\{\mathbf{u}_{+}(\mathbf{r}) \cdot \mathbf{t}\left[\overline{\mathbf{H}}_{\mathrm{L}}(\mathbf{r})\right]-\mathbf{t}_{+}(\mathbf{r}) \cdot \overline{\mathbf{H}}_{\mathrm{L}}(\mathbf{r})\right\}, \\
a_{\mathrm{L}}^{+}=\frac{\mathrm{i} q_{v}}{\rho c_{v}^{2}} \oint_{S} d S\left\{\mathbf{u}_{+}(\mathbf{r}) \cdot \mathbf{t}\left[\overline{\mathbf{J}}_{\mathrm{L}}(\mathbf{r})\right]-\mathbf{t}_{+}(\mathbf{r}) \cdot \overline{\mathbf{J}}_{\mathrm{L}}(\mathbf{r})\right\},
\end{array}
$$

respectively.

The coefficients $a_{\mathrm{L}}^{0}$ and $a_{\mathrm{L}}^{+}$are related to each other through the so-called scattering $T$ matrix, as follows:

$$
a_{\mathrm{L}}^{+}=\sum_{\mathrm{L}^{\prime}} T_{\mathrm{LL}^{\prime}} a_{\mathrm{L}^{\prime}}^{0}
$$

As shown in the Appendix, the $T$ matrix can be obtained, in general, by the solution of a linear system of equations of the form

$$
\mathbf{Q}^{0} \widetilde{\mathbf{T}}=-\mathbf{Q}^{+},
$$

where $\widetilde{\mathbf{T}}$ is the transposed $T$ matrix and $\mathbf{Q}^{0}, \mathbf{Q}^{+}$are appropriate matrices that depend on the specific boundary conditions at the surface of the scatterer. Equation (32) is the basic equation of the EBC method for elastic wave scattering by a particle of arbitrary shape. ${ }^{43-45}$ We note that the $T$ matrix in our spherical-wave basis defined by Eqs. (8), (9), and (10) satisfies the symmetry relation ${ }^{23,54}$

$$
\widetilde{T}_{P \ell m ; P^{\prime} \ell^{\prime} m^{\prime}}=T_{P^{\prime} \ell^{\prime} m^{\prime} ; P \ell m}=\frac{c_{v}^{3}}{c_{v^{\prime}}^{3}} T_{P \ell m ; P^{\prime} \ell^{\prime} m^{\prime}} .
$$

This relation can be used to check the numerical convergence of the $T$ matrix and also to further improve the accuracy of its nondiagonal elements. Moreover, for axis-symmetric particles, $\mathbf{Q}^{0(+)}$ are diagonal in $m$ and we only need to calculate their elements for $m>0$ because $Q_{P \ell-m ; P^{\prime} \ell^{\prime}-m}^{0(+)}=$ $(-1)^{P+P^{\prime}} Q_{P \ell m ; P^{\prime} \ell^{\prime} m}^{0(+)}$. Explicit derivations of the $Q^{0(+)}$ matrix elements for all different material-type combinations of the host and the scatterer can be found in the appendix. We note that, although the spherical-wave expansions of the elastic field are infinite series, it turns out that, if the size of the particle is not much larger than the wavelength, a limited number of partial waves, truncated at $\ell_{\max }$, is sufficient for the description of the scattered field and of the $T$ matrix. However, in order to accurately solve Eq. (32), we must keep matrix elements up to an angular momentum cutoff value $\ell_{\text {cut }} \geqslant \ell_{\text {max }}$. The fact that $\ell_{\text {cut }}$ significantly increases as the shape of the particle deviates from the sphere means that, in order to accurately evaluate the elements of the $T$ matrix of given dimensions, a large number of $Q$-matrix elements are needed. Obviously, for spherical particles $\ell_{\text {cut }}=\ell_{\text {max }}$.

With the help of the $T$ matrix, defined above, one can calculate directly the change in the number of states up to a frequency $\omega$ between the system under consideration (a single scatterer in a host medium) and that of the host medium extending over all space as follows ${ }^{23}$ :

$$
\Delta N(\omega)=\frac{1}{\pi} \operatorname{Im} \ln \operatorname{det}[\mathbf{I}+\mathbf{T}],
$$

where $\mathbf{I}$ is the unit matrix. Of more interest is the difference in the density of states induced by the scatterer, given by $\Delta n(\omega)=d \Delta N(\omega) / d \omega$. On the other hand, the scattering cross section is given by

$$
\sigma_{\mathrm{sc} ; l}=\left|u_{0}\right|^{-2} \sum_{\ell m}\left(\frac{\left|a_{0 \ell m}^{+}\right|^{2}}{q_{l}^{2}}+\frac{q_{l}}{q_{t}} \frac{\left|a_{1 \ell m}^{+}\right|^{2}+\left|a_{2 \ell m}^{+}\right|^{2}}{q_{t}^{2}}\right)
$$

or

$$
\sigma_{\mathrm{sc} ; t}=\left|u_{0}\right|^{-2} \sum_{\ell m}\left(\frac{q_{t}}{q_{l}} \frac{\left|a_{0 \ell m}^{+}\right|^{2}}{q_{l}^{2}}+\frac{\left|a_{1 \ell m}^{+}\right|^{2}+\left|a_{2 \ell m}^{+}\right|^{2}}{q_{t}^{2}}\right)
$$

for a longitudinal or a transverse incident wave, respectively, where $a_{P \ell m}^{+}$are related with the corresponding expansion coefficients $a_{P \ell m}^{0}$ of the incident field, given by Eqs. (12) and (13), through the $T$ matrix according to Eq. (31). It is worth noting that, unlike the case of spherical particles, the cross section depends on the polarization and the direction of propagation of the incident wave. An incident transverse plane wave is termed $p$ polarized if the associated displacement vector oscillates in the plane defined by the propagation direction and the particle axis; it is termed $s$ polarized if the displacement vector oscillates normal to this plane. Obviously, for a spherical particle the polarization of an incident transverse wave is irrelevant. 
A single spherical particle in vacuum exhibits so-called spheroidal and torsional vibrational eigenmodes, of coupled longitudinal-transverse $(P=0,2)$ and purely transverse $(P=1)$ character, respectively. These eigenmodes are denoted by a pair of indices $(i, \ell)$, defining the $\ell$ th multipole mode of the $i$ th radial order ${ }^{55}$ in analogy to the atomic orbitals. When the particle is embedded in a host material, an eigenmode is coupled with the acoustic field in the host and energy is leaking out of the sphere and is radiated away. As a result, the eigenmode acquires a finite lifetime and becomes a resonant mode. This mode is $(2 \ell+1)$-fold degenerate and manifests itself as a peak in the scattering cross section and the density of states. This means that there is a $(2 \ell+1)$-fold degenerate eigenvalue of the $T$ matrix that has a pole in the lower complex-frequency plane at $\omega_{i}-\mathrm{i} \gamma_{i}$ near the real frequency line $\left(0<\gamma_{i} \ll \omega_{i}\right)$, where $\omega_{i}$ is the eigenfrequency and $\gamma_{i}$ the inverse lifetime of the corresponding mode. The change in the density of states in the vicinity of such a mode takes the form of a Lorentzian, ${ }^{56}$

$$
\Delta n(\omega) \approx \frac{2 \ell+1}{\pi} \frac{\gamma_{i}}{\left(\omega-\omega_{i}\right)^{2}+\gamma_{i}^{2}},
$$

occupying an area equal to $2 \ell+1$, centered at $\omega_{i}$ with a half width at half maximum equal to $\gamma_{i}$. In the case of nonspherical particles, there is no clear characterization of multipole modes, since the $T$ matrix is no longer diagonal in angular momentum because of the lack of spherical symmetry, and the eigenmodes can be rigorously classified according to the irreducible representations of the point symmetry group of the particle. ${ }^{57}$ The degeneracy of these modes is equal to the area occupied by the corresponding Lorentzian contribution to the density of states. In what follows we consider oblate spheroidal poly(methyl metacrylate) (PMMA) particles with major axis $A$ and minor axis (axis of revolution) $B$, with an aspect ratio $A: B=4: 3$, in comparison with spherical PMMA particles of equal volume. Therefore, if $S$ is the radius of the spherical particle, the spheroidal particle has $A=2.20 S$ and $B=1.65 \mathrm{~S}$. The point symmetry group of a spheroidal particle is $D_{\infty h}$ (cylindrical symmetry). ${ }^{57,58}$

In Fig. 1 we display the change in the density of states and the corresponding scattering cross section for the given PMMA particle $\left(\rho_{\mathrm{s}}=1190 \mathrm{Kg} / \mathrm{m}^{3}, c_{l s}=2800 \mathrm{~m} / \mathrm{s}, c_{t s}=1400 \mathrm{~m} / \mathrm{s}\right)$ embedded in water $\left(\rho=1000 \mathrm{Kg} / \mathrm{m}^{3}, c_{l}=1480 \mathrm{~m} / \mathrm{s}, c_{t}=0\right)$, in the frequency region about the fundamental (quadrupole) spheroidal eigenmode. We note that, in this case, since only longitudinal waves can subsist in the fluid host medium, only the spheroidal modes of the particle can be excited. Using $\ell_{\max }=5, \ell_{\text {cut }}=7$, and a Gaussian quadrature with seven points for the evaluation of the relevant integrals [Eqs. (A30), (A33), and (A34)] is sufficient to obtain excellent convergence with a relative error less than $10^{-4}$. The fivefold degeneracy of the quadrupole mode of the sphere is removed in the case of the spheroid, giving rise to three Lorentzian contributions to the density of states. Indeed, as shown by broken lines in the top panel of Fig. 1, a Lorentzian fit yields three broad peaks: one at $\omega S / c_{l}=1.741$ associated with a twofold degenerate mode of $E_{2 g}$ symmetry $(m= \pm 2)^{57,58}$ and a lifetime $\gamma^{-1} c_{l} / S \cong 8$, one at $\omega S / c_{l}=1.845$ with the same lifetime $\left(\gamma^{-1} c_{l} / S \cong 8\right)$ associated with a nondegenerate mode of $A_{1 g}$ symmetry $(m=0)$, and one twofold degenerate mode at $\omega S / c_{l}=1.921$
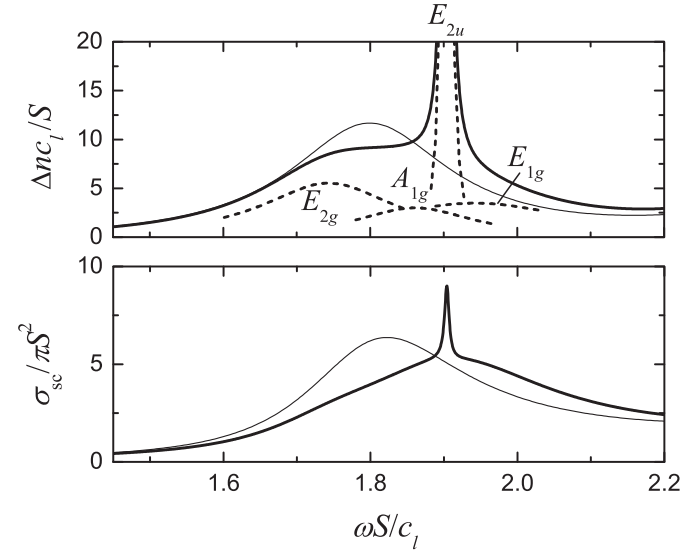

FIG. 1. The change in the density of states of the acoustic field induced by the oblate spheroid PMMA particle under consideration in water (top panel) and the corresponding scattering cross section for incidence at an angle $\theta=45^{\circ}$ with respect to the particle axis (bottom panel). The broken lines show the symmetry deconvolution of $\Delta n$. The thin lines in both diagrams show the results for the corresponding equal-volume sphere of radius $S$.

of $E_{1 g}$ symmetry $(m= \pm 1)$ with a lifetime $\gamma^{-1} c_{l} / S \cong 7$. In addition, there is a sharp peak at $\omega S / c_{l}=1.904$ with a very long lifetime $\left(\gamma^{-1} c_{l} / S \cong 270\right)$. This corresponds to a twofold degenerate mode of $E_{2 u}$ symmetry $(m= \pm 2)$, which originates from a higher-frequency octapole $(\ell=3)$ mode of the sphere. Group theory implies that a (longitudinal) plane wave incident at an angle with respect to the axis of revolution of the spheroid can excite nondegenerate modes of $A_{1 g}$ and $A_{2 u}$ symmetry as well as all doubly degenerate modes, that is, all modes which appear in the frequency region under consideration. However, the modes of short lifetime appear merged into a broad peak in the scattering cross section, as shown in the lower diagram of Fig. 1, and cannot be easily resolved.

We next examine the fundamental modes of the same oblate spheroid PMMA particle embedded in silicon $\left(\rho=2331 \mathrm{Kg} / \mathrm{m}^{3}, c_{l}=8431 \mathrm{~m} / \mathrm{s}, c_{t}=5844 \mathrm{~m} / \mathrm{s}\right)$. Using $\ell_{\max }=3, \ell_{\text {cut }}=5$ and a Gaussian quadrature with five points for the evaluation of the relevant integrals [Eq. (A19)] is sufficient to obtain excellent convergence. In this case, both longitudinal and transverse waves can subsist in the surrounding medium, and all particle modes (spheroidal and torsional) can be excited. A PMMA spherical particle of radius $S$ in silicon exhibits two fundamental dipole modes: a spheroidal one, at $\omega S / c_{l}=0.693$, and a torsional one, at $\omega S / c_{l}=0.738$, as shown by the thin line in the top panel of Fig. 2. The threefold degeneracy of these modes is removed in the case of the spheroid, giving rise to four Lorentzian contributions to the density of states, shown by broken lines in the top panel of Fig. 2. More specifically, the lower-frequency mode splits into a twofold degenerate mode of $E_{1 u}$ symmetry $(m= \pm 1)$ at $\omega S / c_{l}=0.674$ with a lifetime $\gamma^{-1} c_{l} / S \cong 72$ and a nondegenerate mode of $A_{2 u}$ symmetry $(m=0)$ at $\omega S / c_{l}=0.754$ with a lifetime $\gamma^{-1} c_{l} / S \cong 55$. Correspondingly, the higher-frequency mode splits into a nondegenerate mode of $A_{2 g}$ symmetry $(m=0)$ at $\omega S / c_{l}=0.719$ with a lifetime $\gamma^{-1} c_{l} / S \cong 501$ and a twofold 

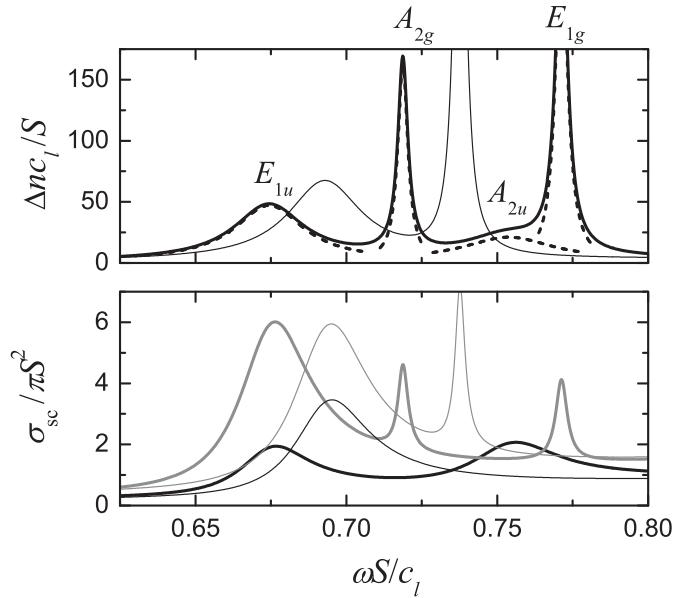

FIG. 2. The change in the density of states of the elastic field induced by the oblate spheroid PMMA particle under consideration in silicon (top panel) and the corresponding scattering cross section for a longitudinal (thick black solid line) and a transverse $s$-polarized (thick gray solid line) wave incident at an angle $\theta=45^{\circ}$ with respect to the particle axis (bottom panel). The broken lines show the symmetry deconvolution of $\Delta n$. The thin lines in both diagrams show the results for the corresponding equal-volume sphere of radius $S$.

degenerate mode of $E_{1 g}$ symmetry $(m= \pm 1)$ at $\omega S / c_{l}=$ 0.771 with a lifetime $\gamma^{-1} c_{l} / S \cong 442$. A transverse $s$-polarized plane wave incident at an angle with respect to the axis of revolution of the spheroid excites the modes of $E_{1 u}, A_{2 g}$, and $E_{1 g}$ symmetry, as we can see in the bottom panel of Fig. 2 (thick black solid line). It cannot excite the $A_{2 u}$ mode for symmetry reasons. On the contrary, a longitudinal plane wave incident at an angle with respect to the axis of revolution of the spheroid excites the modes of $E_{1 u}$ and $A_{2 u}$ symmetry as can be seen in the lower diagram of Fig. 2 (thick gray solid line). According to group theory, such an incident wave should also excite the $E_{1 g}$ mode. However, the coupling of the given external field with this mode is so weak that no excitation is practically observed in the corresponding scattering cross section.

\section{PHONONIC CRYSTAL SLABS}

We consider a layer of nonoverlapping particles, at $z=0$, centered at the sites $\mathbf{R}_{n}$ of a 2D lattice. Let a plane wave, described by Eq. (7), be incident on this layer. Because of the 2D periodicity, we write the component of the wave vector of the incident wave parallel to the layer, $\mathbf{q}_{\|}$, as $\mathbf{q}_{\|}=\mathbf{k}_{\|}+\mathbf{g}^{\prime}$, where $\mathbf{k}_{\|}$, the reduced wave vector in the surface Brillouin zone (SBZ), is a conserved quantity in the scattering process and $\mathbf{g}^{\prime}$ is a reciprocal vector of the given lattice. Therefore, the wave vector of the incident wave has the form $\mathbf{K}_{\mathbf{g}^{\prime} v^{\prime}}^{ \pm}=$ $\mathbf{k}_{\|}+\mathbf{g}^{\prime} \pm\left[q_{v^{\prime}}^{2}-\left(\mathbf{k}_{\|}+\mathbf{g}^{\prime}\right)^{2}\right]^{1 / 2} \hat{\mathbf{e}}_{z}$, where $\hat{\mathbf{e}}_{z}$ is the unit vector along the $z$ axis and the + or - sign refers to incidence from the left $(z<0)$ or from the right $(z>0)$. This implies the following form for the corresponding displacement vector:

$$
\mathbf{u}_{\text {in }}(\mathbf{r})=\left[u_{\text {in }}\right]_{\mathbf{g}^{\prime} \mathbf{p}^{\prime}}^{s^{\prime}} \exp \left(\mathrm{i} \mathbf{K}_{\mathbf{g}^{\prime} v^{\prime}}^{s^{\prime}} \cdot \mathbf{r}\right) \hat{\mathbf{e}}_{\mathrm{p}^{\prime}}\left(\mathbf{K}_{\mathbf{g}^{\prime} v^{\prime}}^{s^{\prime}}\right)
$$

where $s^{\prime}=+$ or - . For $v^{\prime}=l$, only the radial $\left(\mathrm{p}^{\prime}=1\right)$ component of the displacement vector is different from zero, $\hat{\mathbf{e}}_{1}$ being the radial unit vector along the direction of $\mathbf{K}_{\mathbf{g}^{\prime} l}^{s^{\prime}}$; and for $v^{\prime}=t$, only the polar $\left(\mathrm{p}^{\prime}=2\right)$ and azimuthal $\left(\mathrm{p}^{\prime}=3\right)$ components of the displacement vector are different from zero, $\hat{\mathbf{e}}_{2}$ and $\hat{\mathbf{e}}_{3}$ being the polar and azimuthal unit vectors, respectively, which are, of course, perpendicular to $\mathbf{K}_{\mathbf{g}^{\prime} t}^{s^{\prime}}$. According to Eqs. (12) and (13) the coefficients $a_{\mathrm{L}}^{0}$ in the regular spherical waves expansion (26) of the incident plane wave (38) are $a_{\mathrm{L}}^{0}=A_{\mathrm{L} ; p^{\prime}}^{0}\left(\mathbf{K}_{\mathbf{g}^{\prime} v^{\prime}}^{s^{\prime}}\right)\left[u_{\mathrm{in}}\right]_{\mathbf{g}^{\prime} \mathbf{p}^{\prime}}^{s^{\prime}}$.

Because of the 2D periodicity of the plane of particles, for an incident wave given by Eq. (38) the scattered wave has the form

$$
\mathbf{u}_{\mathrm{sc}}(\mathbf{r})=\sum_{\mathbf{R}_{n}} \exp \left(\mathrm{i} \mathbf{k}_{\|} \cdot \mathbf{R}_{n}\right) \sum_{\mathrm{L}} b_{\mathrm{L}}^{+} \mathbf{H}_{\mathrm{L}}\left(\mathbf{r}_{n}\right),
$$

where $\mathbf{r}_{n}=\mathbf{r}-\mathbf{R}_{n}$. Writing the coefficients $b_{\mathrm{L}}^{+}$, which depend linearly on the amplitude of the incident wave, in the form $b_{\mathrm{L}}^{+}=B_{\mathrm{L} ; \mathrm{p}^{\prime}}^{+}\left(\mathbf{K}_{\mathbf{g}^{\prime} v^{\prime}}^{s^{\prime}}\right)\left[u_{\mathrm{in}}\right]_{\mathbf{g}^{\prime} \mathrm{p}^{\prime}}^{s^{\prime}}$, we obtain $\mathbf{B}_{\mathrm{L}}^{+}$in terms of $\mathbf{A}_{\mathrm{L}}^{0}$ by solving the following system of linear equations ${ }^{6}$ :

$$
\begin{gathered}
\sum_{\mathrm{L}^{\prime}}\left[\delta_{\mathrm{LL}^{\prime}}-\sum_{\mathrm{L}^{\prime \prime}} T_{\mathrm{LL}^{\prime \prime}} \Omega_{\mathrm{L}^{\prime \prime} \mathrm{L}^{\prime}}\right] B_{\mathrm{L}^{\prime} ; \mathrm{p}^{\prime}}^{+}\left(\mathbf{K}_{\mathbf{g}^{\prime} v^{\prime}}^{s^{\prime}}\right) \\
=\sum_{\mathrm{L}^{\prime}} T_{\mathrm{LL}^{\prime}} A_{\mathrm{L}^{\prime} ; \mathrm{p}^{\prime}}^{0}\left(\mathbf{K}_{\mathbf{g}^{\prime} v^{\prime}}^{s^{\prime}}\right), \quad \forall \mathrm{L} .
\end{gathered}
$$

Explicit expressions for the propagator matrix elements $\Omega_{\mathrm{LL}^{\prime}}$ are given in Ref. 6. We note that, for particles of cylindrical symmetry, the elements of both the $\Omega$ and the $T$ matrices, $\Omega_{P \ell m ; P^{\prime} \ell^{\prime} m^{\prime}}$ and $T_{P \ell m ; P^{\prime} \ell^{\prime} m^{\prime}}$, respectively, vanish identically, unless $P+\ell+m+P^{\prime}+\ell^{\prime}+m^{\prime}$ is an even number. Therefore, in this case the linear system described by Eq. (40) can be reduced into two independent subsystems, as for spherical particles, ${ }^{6}$ thus increasing the computational efficiency.

With the help of $\mathbf{B}_{\mathrm{L}}^{+}$one can define the scattering $S$ matrix of the plane of scatterers, which transforms the incident into the outgoing wave field in the spg basis, in exactly the same manner as for a plane of spherical particles [it is the $M$ matrix defined in Eq. (4.24) of Ref. 4]. The scattering $S$ matrix of a multilayer slab is obtained by combining appropriate transmission and reflection matrices of the component layers in the usual manner, ${ }^{22}$ and this procedure is also independent on the shape of the scatterers. The ratio of the transmitted or reflected energy flux to the energy flux associated with the incident wave defines the transmittance, $\mathcal{T}$, or reflectance, $\mathcal{R}$, of the slab, respectively. ${ }^{22}$ If absorption is present, it can be calculated from the requirement of energy conservation: $\mathcal{A}=1-\mathcal{T}-\mathcal{R}$.

The $S$ matrix can provide, directly, the $\mathbf{k}_{\|}$-resolved change in the number of states, up to a frequency $\omega$, induced by the $\operatorname{slab}^{22,23}$

$$
\Delta N\left(\omega, \mathbf{k}_{\|}\right)=\frac{1}{2 \pi} \operatorname{Im} \ln \operatorname{det} \mathbf{S},
$$

which defines the corresponding change in the density of states $\Delta n\left(\omega, \mathbf{k}_{\|}\right)=\frac{\partial}{\partial \omega} \Delta N\left(\omega, \mathbf{k}_{\|}\right)$. The $S$ matrix is a unitary matrix because of flux conservation while the causality condition implies that its eigenvalues are analytic functions in the upper complex-frequency half plane. However, as in the case of the $T$ matrix, these eigenvalues may have poles in the lower half plane at $\omega_{i}-\mathrm{i} \gamma_{i}, \gamma_{i}>0$, corresponding to zeros at $\omega_{i}+\mathrm{i} \gamma_{i}$, 
which are associated with resonant modes. The contribution of a simple pole to the $\mathbf{k}_{\|}$-resolved density of states has the form of a Lorentzian resonance, ${ }^{22}$

$$
\Delta n\left(\omega, \mathbf{k}_{\|}\right) \approx \frac{1}{\pi} \frac{\gamma_{i}}{\left(\omega-\omega_{i}\right)^{2}+\gamma_{i}^{2}},
$$

analogous to Eq. (37). The resonant modes of a phononic crystal slab manifest themselves as Fano-like resonance structures also in the corresponding transmission spectrum, which takes the form ${ }^{22}$

$$
\mathcal{T} \simeq \cos ^{2}\left( \pm \delta_{1} \pm \delta_{2} \cdots-\phi\right),
$$

with $\sin \left[\delta_{i}(\omega)\right]=\gamma_{i} /\left[\left(\omega-\omega_{i}\right)^{2}+\gamma_{i}^{2}\right]^{1 / 2}$ and $\cos \left[\delta_{i}(\omega)\right]=$ $\left(\omega_{i}-\omega\right) /\left[\left(\omega-\omega_{i}\right)^{2}+\gamma_{i}^{2}\right]^{1 / 2}$. The sign + or - in $\delta_{i}$ and the adjustable phase parameter $\phi$ can be determined by fitting the actual transmission spectrum to Eq. (43).

Assembling particles on a 2D lattice, we expect that their resonant modes will form relatively narrow bands about the corresponding eigenfrequencies of the single particle. At the symmetry points and along the symmetry lines of the SBZ, these bands can be classified in terms of the irreducible representations of the point group of the corresponding wave vector. For example, for a square lattice and $\mathbf{k}_{\|}=(0,0)$ ( $\bar{\Gamma}$ point $)$, the modes have the symmetry of the irreducible representations of the $D_{4 h}$ group: $X_{1}, X_{2}, X_{3}, X_{4}, X_{5}, X_{1^{\prime}}$, $X_{2^{\prime}}, X_{3^{\prime}}, X_{4^{\prime}}, X_{5^{\prime}}{ }^{58}$ The states of $X_{5}$ and $X_{5^{\prime}}$ symmetry are doubly degenerate while the rest are nondegenerate. According to a group-theory analysis, ${ }^{57,58}$ the nondegenerate modes of $A_{1 g}, A_{2 g}, A_{1 u}$, and $A_{2 u}$ symmetry of a spheroidal particle give at the $\bar{\Gamma}$ point nondegenerate modes of $X_{1}, X_{4}, X_{1^{\prime}}$, and $X_{4^{\prime}}$ symmetry, respectively. Similarly, the doubly degenerate $E_{1 g}$ and $E_{1 u}$ modes give doubly degenerate modes of $X_{5}$ and $X_{5^{\prime}}$ symmetry, respectively, while a $E_{2 g}$ or a $E_{2 u}$ mode splits into two nondegenerate modes $\left(X_{2}\right.$ and $X_{3}$ or $X_{2^{\prime}}$ and $X_{3^{\prime}}$, respectively).

Let us consider a square array, with lattice constant $a$, of oblate spheroidal PMMA particles $(A=0.88 a, B=0.66 a)$ in water. In Fig. 3 we depict the change in the density of states of the system with respect to water, for $\mathbf{k}_{\|}=(0,0)$, and the corresponding transmittance, in the frequency region about the fundamental quadrupole mode of a PMMA sphere of equal volume $(S=0.4 a)$. In this case, excellent convergence is obtained by truncating the spherical-wave expansions at $\ell_{\max }=8$ and $\ell_{\text {cut }}=8$ and using a Gaussian quadrature with eight points for the relevant integrals (see Sec. III), while in the plane-wave expansions only the $\mathbf{g}=(0,0)$ reciprocal lattice vector that defines a propagating wave should be taken into account. The symmetry of a longitudinal plane acoustic wave propagating in the water host, normal to the plane of particles $\left[\mathbf{q}_{\|}=(0,0)\right]$, is compatible with the $X_{1}$ and $X_{4^{\prime}}$ symmetries, so only modes of the plane of particles of these symmetries can be excited by an externally incident wave. The modes of different symmetry are inactive and decay exponentially to zero away from the plane of particles on either side of it. The inactive modes (eigenmodes) show up as $\delta$ functions and the active ones as Lorentzian resonances in the corresponding density of states. As can be seen in the top panel of Fig. 3, the modes of the single particle give, for $\mathbf{k}_{\|}=(0,0)$, eigenmodes at $\omega a / c_{l}=3.959\left(X_{2}\right), 4.712\left(X_{3^{\prime}}\right), 4.786\left(X_{2^{\prime}}\right), 4.877\left(X_{3}\right)$, and

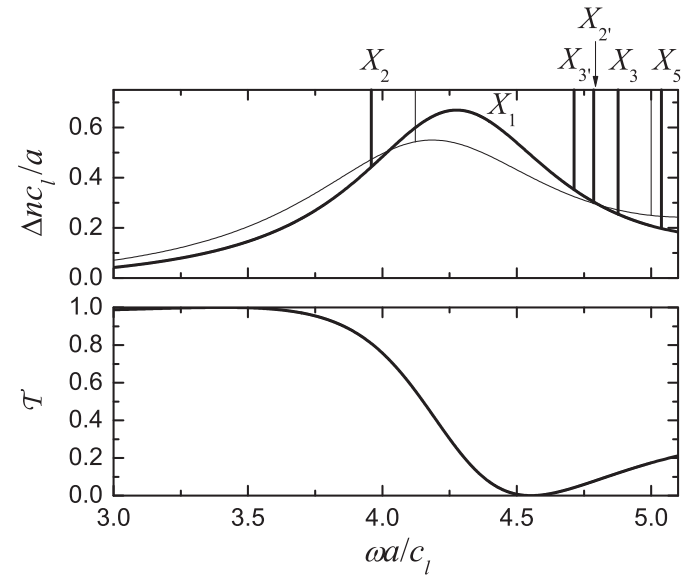

FIG. 3. (Top) The change in the $\mathbf{k}_{\|}$-resolved density of states of water due to the presence of a square array, with lattice constant $a$, of PMMA oblate spheroidal particles $(A=0.88 a, B=0.66 a)$ for $\mathbf{k}_{\|}=(0,0)$. The thin line shows the corresponding results for equalvolume spheres of radius $S=0.4 a$. (Bottom) The transmittance of an acoustic (longitudinal) wave incident normally on the plane of spheroidal particles.

$5.038\left(X_{5}\right)$ and a $X_{1}$ resonant mode at $\omega_{1} a / c_{l}=4.281$ with a lifetime $\gamma^{-1} c_{l} / a \cong 2$. In the bottom panel of Fig. 3 we display the corresponding transmission spectrum. It is characterized by a resonance structure and is very well described by the function $\mathcal{T}=\cos ^{2}\left(\delta_{1}-\phi\right)$ with $\phi=30^{\circ} 12^{\prime}$, according to Eq. (43).

For $\mathbf{k}_{\|}=\left(k_{x}, 0\right)$, the appropriate point group is $C_{2 v}$, which has four one-dimensional irreducible representations: $Z_{1}, Z_{2}$, $Z_{3}, Z_{4} \cdot{ }^{58}$ Only $Z_{1}$ and $Z_{4}$ are compatible with a longitudinal elastic plane wave incident on the plane of particles with the given $\mathbf{k}_{\|}$. Figure 4 shows the modes of the plane of particles for $\mathbf{k}_{\|}=(0.2 \pi / a, 0)$. They manifest themselves as Lorentzian peaks of area equal to unity in the density of states and as Fanolike resonance structures in the corresponding transmission spectrum, which is very well reproduced by Eq. (43) using a single fitting parameter $\phi$. The $X_{2}$ and $X_{3^{\prime}}$ eigenmodes for $\mathbf{k}_{\|}=(0,0)$ become resonant $Z_{1}$ and $Z_{4}$ modes at $\omega a / c_{l}=$ 3.980 and 4.703 with very long lifetimes $\left(\gamma^{-1} c_{l} / a \cong 1212\right.$ and 760, respectively). The $X_{1}$ resonant mode acquires the $Z_{1}$ symmetry and is slightly shifted at $\omega a / c_{l}=4.306$. Because of its very short lifetime $\left(\gamma^{-1} c_{l} / a \cong 2\right)$ it is hardly discernible

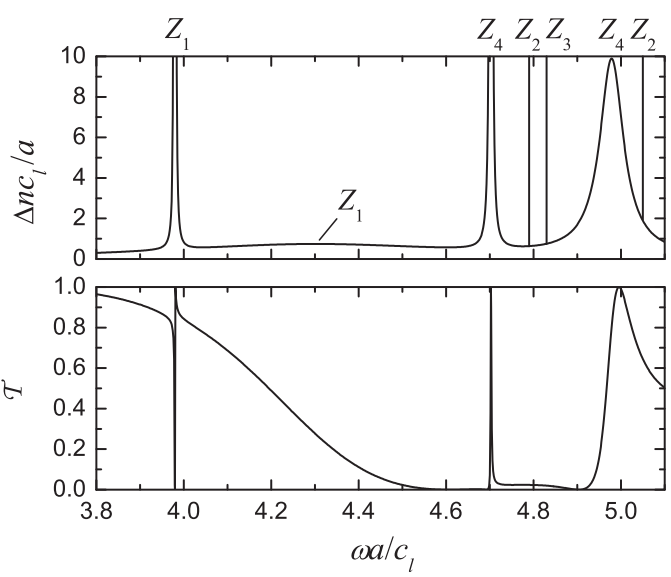

FIG. 4. The same as in Fig. 3, for $\mathbf{k}_{\|}=(0.2 \pi / a, 0)$. 


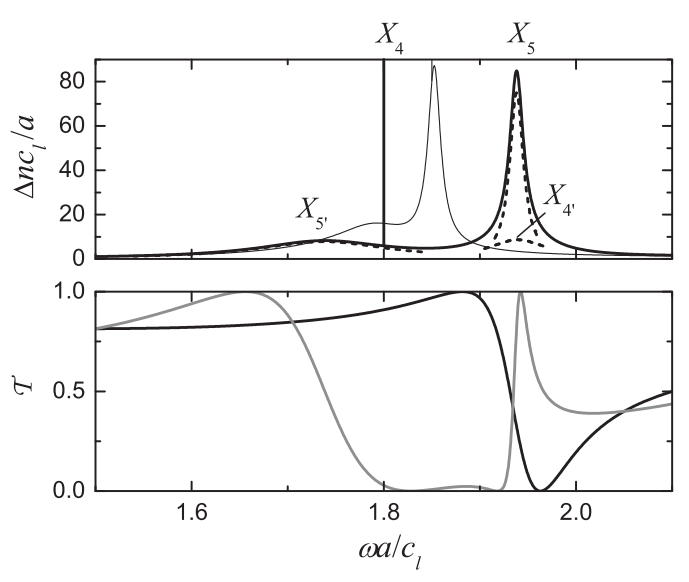

FIG. 5. (Top) The change in the $\mathbf{k}_{\|}$-resolved density of states of a silicon matrix due to the presence of a square array, with lattice constant $a$, of PMMA oblate spheroidal particles ( $A=0.88 a$, $B=0.66 a)$ for $\mathbf{k}_{\|}=(0,0)$. The broken lines show the symmetry deconvolution of $\Delta n$. The thin line shows the corresponding results for equal-volume spheres. (Bottom) The transmittance of a longitudinal (black solid line) and a transverse (gray solid line) elastic wave incident normally on the given plane of spheroidal particles.

in the scale of the figure. The $X_{2^{\prime}}$ and $X_{3}$ eigenmodes remain true eigenmodes with infinite lifetime at $\omega a / c_{l}=4.786\left(Z_{2}\right)$ and $4.828\left(Z_{3}\right)$, respectively. Finally, the $X_{5}$ eigenmode splits into a $Z_{4}$ resonant mode at $\omega a / c_{l}=4.979$ with a lifetime $\gamma^{-1} c_{l} / a \cong 30$ and a $Z_{2}$ eigenmode at $\omega a / c_{l}=5.046$.

We next consider the given square array of PMMA oblate spheroidal particles embedded in silicon. Figure 5 depicts the change in the density of states of the system with respect to silicon, for $\mathbf{k}_{\|}=(0,0)$, and the corresponding transmission spectrum, in the frequency region about the fundamental dipole modes of the PMMA particles. In this case, excellent convergence is attained by truncating the spherical-wave expansions at $\ell_{\max }=8$ and $\ell_{\text {cut }}=8$ and using a Gaussian quadrature with 10 points for the relevant integrals (see Sec. III), while in the plane-wave expansions only the $\mathbf{g}=(0,0)$ reciprocal lattice vector defines a propagating wave and should be taken into account. We should remember that a longitudinal plane acoustic wave propagating in the host region normal to the given layer has a symmetry compatible with $X_{1}$ and $X_{4^{\prime}}$. Similarly, the symmetry of a corresponding transverse wave is compatible with $X_{5}$ and $X_{5^{\prime}}$. Therefore, only the $X_{4}$ mode at $\omega a / c_{l}=1.804$ is an eigenmode of the layer with infinite lifetime and shows up as a $\delta$ function in the density of states (top panel of Fig. 5). The $X_{4^{\prime}}$ resonant mode at $\omega a / c_{l}=1.940$ with a lifetime $\gamma^{-1} c_{l} / a \cong 27$ can be excited by a longitudinal wave (see the black solid line in the bottom panel of Fig. 5). Correspondingly, the $X_{5^{\prime}}$ resonant mode at $\omega a / c_{l}=1.737$ with $\gamma^{-1} c_{l} / a \cong 12$ and the $X_{5}$ resonant mode at $\omega a / c_{l}=1.938$ with $\gamma^{-1} c_{l} / a \cong 118$ manifest themselves as Fano-like resonance structures in the transmittance of a transverse wave (see gray solid line in the bottom panel of Fig. 5).

For $\mathbf{k}_{\|}=(0.2 \pi / a, 0)$ (see Fig. 6), the symmetry of the slab modes is lower $\left(C_{2 v}\right.$ point group). In this case, the $X_{5^{\prime}}$ resonant mode splits into a $Z_{3}$ resonant mode at $\omega a / c_{l}=1.733$ with $\gamma^{-1} c_{l} / a \cong 14$ and a $Z_{1}$ resonant mode at $\omega a / c_{l}=1.740$ with

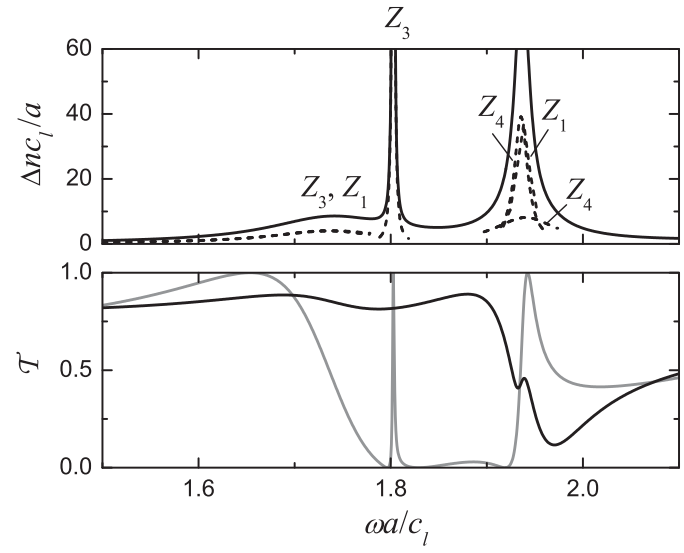

FIG. 6. The same as in Fig. 5 for $\mathbf{k}_{\|}=(0.2 \pi / a, 0)$. The transmittance of the transverse wave is for $s$ polarization.

$\gamma^{-1} c_{l} / a \cong 12$. The $X_{4}$ eigenmode becomes a resonant $Z_{3}$ mode at $\omega a / c_{l}=1.803$ with $\gamma^{-1} c_{l} / a \cong 893$. The $X_{4^{\prime}}$ resonant mode is practically unshifted and acquires the $Z_{4}$ symmetry with $\gamma^{-1} c_{l} / a \cong 25$. Finally, the $X_{5}$ resonant mode splits into a $Z_{4}$ resonant mode at $\omega a / c_{l}=1.935$ with $\gamma^{-1} c_{l} / a \cong 122$ and a $Z_{2}$ resonant mode at $\omega a / c_{l}=1.938$ with $\gamma^{-1} c_{l} / a \cong 116$. The $Z_{2}$ and $Z_{3}$ modes manifest themselves as Fano-like resonance structures in the transmission spectrum of an $s$ polarized transverse elastic wave, while the $Z_{1}$ and $Z_{4}$ modes can be probed by an incident longitudinal (or $p$-polarized transverse, not shown here) elastic wave, in agreement with group theoretical arguments.

\section{PHONONIC CRYSTALS}

Within the LMS method, an (infinite) phononic crystal is viewed as a sequence of identical slabs parallel to a given crystallographic plane, which is taken to be the $x-y$ plane, extending over all space from $z=-\infty$ to $z=+\infty$. The unit slab may consist of any number of layers, provided they have the same 2D periodicity. Applying the Bloch condition for the wave field in the host region between two consecutive unit slabs leads to an eigenvalue equation, which gives the $z$ component of the Bloch wave vector, $k_{z}$, for given $\omega$ and $\mathbf{k}_{\|}$, in exactly the same manner as for a crystal of spherical particles. $^{6}$

As a first example, we consider a simple cubic (sc) crystal, with lattice constant $a$, of PMMA oblate spheroidal particles, with $A=0.88 a$ and $B=0.66 a$, in water and view the crystal as a sequence of (001) planes of particles, as those described in Sec. IV. In this case we need 21 reciprocal lattice vectors to accurately describe multiple scattering between the layers. In the left panel of Fig. 7 we show the phononic band structure of this crystal along its [001] direction, that is, for $\mathbf{k}_{\|}=(0,0)$. The symmetry of the bands along this direction is that of the $C_{4 v}$ group: $\Delta_{1}, \Delta_{2}, \Delta_{1^{\prime}}, \Delta_{2^{\prime}}, \Delta_{5} \cdot{ }^{58}$ The $\Delta_{1}, \Delta_{2}, \Delta_{1^{\prime}}, \Delta_{2^{\prime}}$ bands are nondegenerate and $\Delta_{5}$ is doubly degenerate. We note that the (001) surface of the given crystal is a plane of mirror symmetry and, therefore, the frequency bands appear in pairs: $k_{z}\left(\omega, \mathbf{k}_{\|}\right)$and $-k_{z}\left(\omega, \mathbf{k}_{\|}\right)$. For this reason, in Fig. 7 , we show the bands only for positive $k_{z}$. At low frequencies we obtain a linear effective-medium acoustic band of $\Delta_{1}$ symmetry, 


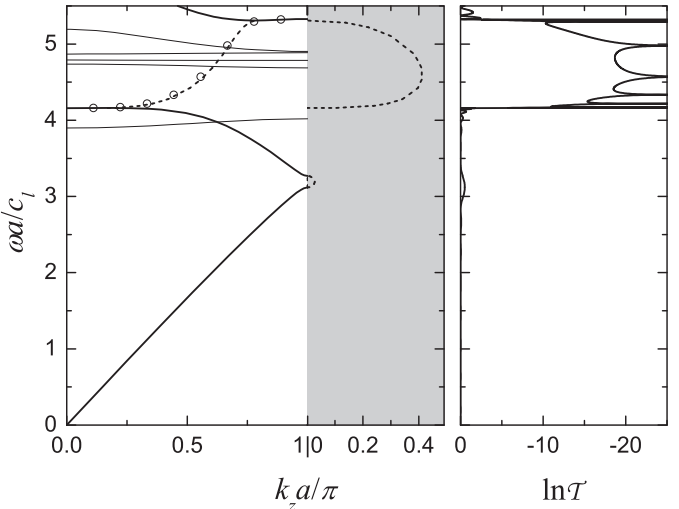

FIG. 7. (Left) The phononic band structure of an infinite sc crystal, with lattice constant $a$, of PMMA oblate spheroidal particles, with $A=0.88 a$ and $B=0.66 a$, in water, along its [001] direction. Over the frequency gap, we show by broken lines the complex bands with the smallest-in-magnitude imaginary part of $k_{z}$ of the appropriate $\left(\Delta_{1}\right)$ symmetry. The imaginary part is shown in the shaded region. (Right) Transmittance at normal incidence of a slab of eight (001) planes of the above crystal.

which is folded into the first Brillouin zone and a Bragg gap opens up about $\omega a / c_{l}=3.2$. At higher frequencies, this band crosses narrow bands that originate from the resonant modes of the individual PMMA spheroids weakly interacting between them. The $\Delta_{1}$ resonance band, which is formed from the $A_{1 g}$ particle modes, hybridizes strongly with the extended band and a sizable frequency gap opens up about $\omega a / c_{l}=4.2$. The other narrow bands, of $\Delta_{2}$ (about $\omega a / c_{l}=4.0$ and 4.7), $\Delta_{2^{\prime}}$ (about $\omega a / c_{l}=4.8$ and 4.9 ), and $\Delta_{5}$ (about $\omega a / c_{l}=5.0$ ) symmetry, arise from an apparently weak interaction between eigenmodes that are confined within the individual layers (inactive modes). These bands cannot couple to a compressional incident wave and are termed deaf bands. Within the frequency regions where there are no active bands of $\Delta_{1}$ symmetry, we show the complex $\Delta_{1}$ bands with the smallest-in-magnitude imaginary part of $k_{z}$, which determines the attenuation of a wave incident on the crystal with $\mathbf{k}_{\|}=(0,0)$ over these regions. This is demonstrated in the right panel of Fig. 7, where we depict the transmittance for a wave incident normally on a slab consisting of $N_{\mathrm{L}}=$ 8 (001) planes of the given crystal. Within frequency regions where $\Delta_{1}$ bands exist, we have strong transmission. Otherwise, the transmission is vanishingly small. Interestingly, in the gap region, the transmission is related also to the real part of the $\Delta_{1}$ complex band extending from $\omega a / c_{l}=4.16$ to $\omega a / c_{l}=5.31$. The observed sharp dips in the transmittance are associated with resonances of the slab that appear at frequencies along the real-frequency line corresponding to $\operatorname{Re}\left[k_{z}\right] a / \pi=\kappa /\left(N_{\mathrm{L}}+\right.$ 1 ), with $\kappa=1,2, \ldots, N_{\mathrm{L}}$, where $N_{\mathrm{L}}=8$ is the number of planes in the slab, as shown by the open circles in the left panel of Fig. 7. This implies that the number of resonance dips over the gap region increases with the thickness $N_{\mathrm{L}}$ of the slab.

We next discuss the phononic band structure along the [001] direction of a sc crystal, with lattice constant $a$, of PMMA oblate spheroidal particles, with $A=0.88 a$ and $B=0.66 a$, in silicon. We employ 21 reciprocal lattice vectors to accurately describe multiple scattering between the layers. In this case there are both longitudinal and transverse waves

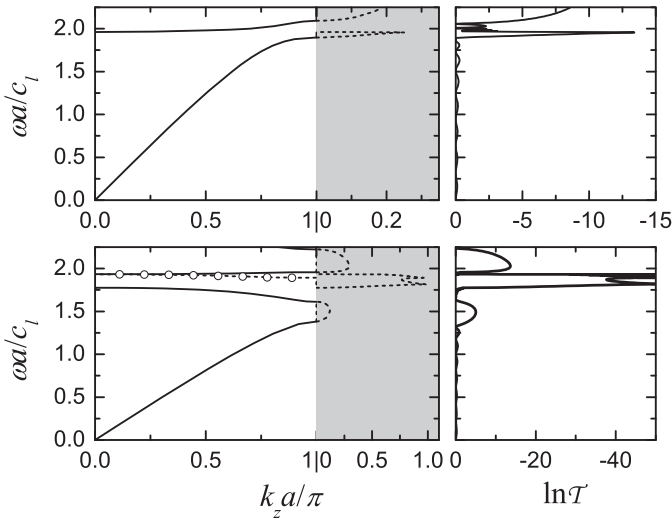

FIG. 8. (Left panels) The phononic band structure of an infinite sc crystal, with lattice constant $a$, of PMMA oblate spheroidal particles, with $A=0.88 a$ and $B=0.66 a$, in silicon, along its [001] direction, for the $\Delta_{1}$ (top) and $\Delta_{5}$ (bottom) modes. Over the frequency gaps, we show by broken lines the corresponding complex bands with the smallest in magnitude imaginary part of $k_{z}$. The imaginary part is shown in the shaded region. (Right panels) Transmittance of a slab of eight (001) planes of the above crystal at normal incidence for longitudinal (top) and transverse (bottom) incident waves.

traveling in the crystal, which can be treated separately. In the upper diagram of Fig. 8 we present the (longitudinal) modes of $\Delta_{1}$ symmetry. We obtain an extended acoustic band which interacts strongly with the $\Delta_{1}$ resonance band that originates from the $A_{2 u}$ modes of the individual spheroids and a hybridization gap opens up at $\omega a / c_{l}=1.90$. In the lower diagram of Fig. 8 we depict the (transverse) modes of $\Delta_{5}$ symmetry. The extended acoustic band is folded within the first Brillouin zone and a Bragg gap opens up at $\omega a / c_{l}=1.38$. Furthermore, hybridization gaps are formed by the interaction of this extended band with the $\Delta_{5}$ flat bands originating from the $E_{1 u}$ and $E_{1 g}$ modes of the individual particles. These hybridization gaps superimpose with the Bragg gap and, as a result, we obtain two flat bands of localized modes within a large gap. Such a behavior is not observed in the case of the corresponding spherical particles. In the gap regions, when the appropriate complex band with the smallest-in-magnitude imaginary part of $k_{z}$ has $\operatorname{Re}\left[k_{z}\right] \neq 0, \pi / a$, the transmittance exhibits sharp dips at corresponding frequencies shown by the open circles in the bottom left panel of Fig. 8, as discussed in relation to Fig. 7. Finally, we note that there is also a nondegenerate flat band of $\Delta_{1^{\prime}}$ symmetry about $\omega a / c_{l}=1.80$, originating from the $X_{4}$ modes of the individual (001) layers of particles, weakly interacting between them. This is an inactive (deaf) band, which cannot be excited by a longitudinal or a transverse wave and is not shown in the diagrams of Fig. 7.

\section{CONCLUSIONS}

In summary, we presented an extension of the LMS method for phononic crystals consisting of nonspherical particles in a homogeneous host medium using the EBC approach to describe the individual particles and showed that the method retains its efficiency and accuracy, at least in cases where the shape of the particles does not deviate strongly from the sphere. The applicability of the method is demonstrated on specific 
examples of 2D and 3D periodic assemblies of spheroidal polymer particles in a fluid and a solid environment. The symmetry of the modes of the elastic field in the different cases is analyzed in terms of group theory, yielding a consistent interpretation of the results. A detailed analysis of transmission spectra of such finite phononic crystal slabs by reference to corresponding complex-band-structure and density-of-states diagrams corroborates that nonspherical particles provide an additional degree of freedom for tailoring the modes of the elastic field. There are many applications expected from the effect of distortion of spherical scatterers, an example being single-mode acoustic waveguides based on weakly coupled defects in a phononic band gap crystal. The high degeneracy of the modes of a single spherical defect would imply a large number of bands in the same frequency region within the gap for a chain of such defects. By deforming the spherical shape, a single band could split off, thus ensuring single-mode operation in a given range of frequencies. Such applications have already been reported for coupled-cavity optical waveguides. ${ }^{59}$ Moreover, nonspherical particles are versatile building units for designing functional phononic structures for polarization-selective applications as well as novel acousto-optic devices. ${ }^{60}$

\section{ACKNOWLEDGMENTS}

The authors are grateful to S. Ivansson for helpful discussions. The research leading to these results has received funding from the European Community's Seventh Framework Programme (FP7/2007-2013) under Grant Agreement No. 233883 (TAILPHOX).

\section{APPENDIX}

In this appendix we derive explicit expressions for the evaluation of the $Q^{0(+)}$ matrices that lead to the calculation of the scattering $T$ matrix according to Eq. (32). Though similar formulas can be found in the literature, we thought it necessary to include this appendix in order to provide the equations in a compact form, compatible to our formalism, for material combinations of any type, fluid or solid, including also the limiting cases of the void and the perfectly rigid body. We consider scatterers that remain invariant under any rotation around the $z$ axis (axis-symmetric scatterers). Therefore, the surface points are given by $\mathbf{r}=r(\theta) \hat{\mathbf{r}}$ and a corresponding normal vector, which must be parallel to $(\partial \mathbf{r} / \partial \theta) \times(\partial \mathbf{r} / \partial \phi)$, is defined as

$$
\mathbf{n} \equiv \hat{\mathbf{r}}-\frac{r^{\prime}(\theta)}{r(\theta)} \hat{\boldsymbol{\theta}},
$$

and has a length

$$
n=\sqrt{1+\left[\frac{r^{\prime}(\theta)}{r(\theta)}\right]^{2}}
$$

Thus, the surface normal unit vector is $\hat{\mathbf{n}}=\mathbf{n} / n$ and the area element becomes $d S=n r^{2} \sin \theta d \theta d \phi$.

For the calculation of $a_{\mathrm{L}}^{0}$ and $a_{\mathrm{L}}^{+}$, on the basis of Eqs. (29) and (30), one needs to evaluate scalar products of vector spherical waves and associated surface tractions at the surface of the scatterer. Since the vector spherical waves, $\mathbf{F}_{\mathrm{L}}(r, \theta, \phi)$, at the surface points of an axis-symmetric scatterer $[\mathbf{r}=r(\theta) \hat{\mathbf{r}}]$ depend on the azimuthal angle $\phi$ through the factor $\exp (\mathrm{i} m \phi)$, as can be seen from their explicit expressions, the $\phi$ integration in Eqs. (29) and (30) can be carried out analytically. For this purpose, we write $\mathbf{F}_{\mathrm{L}}(r, \theta, \phi)=\left[F_{\mathrm{L} ; 1}(r, \theta) \hat{\mathbf{r}}+F_{\mathrm{L} ; 2}(r, \theta) \hat{\boldsymbol{\theta}}-\right.$ $\left.\mathrm{i} F_{\mathrm{L} ; 3}(r, \theta) \hat{\boldsymbol{\phi}}\right] \exp (\mathrm{im} \phi)$, which implies $\overline{\mathbf{F}}_{\mathrm{L}}(r, \theta, \phi)=$ $\left[F_{\mathrm{L} ; 1}(r, \theta) \hat{\mathbf{r}}+F_{\mathrm{L} ; 2}(r, \theta) \hat{\boldsymbol{\theta}}+\mathrm{i} F_{\mathrm{L} ; 3}(r, \theta) \hat{\boldsymbol{\phi}}\right] \exp (-\mathrm{i} m \phi)$.

Therefore, the scalar product of two vector spherical waves, $\mathbf{F}_{\mathrm{L}}^{(1)}(r, \theta, \phi)$ and $\overline{\mathbf{F}}_{\mathrm{L}^{\prime}}^{(2)}(r, \theta, \phi)$, has the form

$$
\mathbf{F}_{\mathrm{L}}^{(1)}(r, \theta, \phi) \cdot \overline{\mathbf{F}}_{\mathrm{L}^{\prime}}^{(2)}(r, \theta, \phi)=\mathbf{F}_{\mathrm{L}}^{(1)}(r, \theta) \cdot \mathbf{F}_{\mathrm{L}^{\prime}}^{(2)}(r, \theta) \exp \left[\mathrm{i}\left(m-m^{\prime}\right) \phi\right]
$$

where

$$
\begin{gathered}
\mathbf{F}_{0 \ell m}(r, \theta)=f_{\ell}^{\prime}\left(x_{l}\right) Y_{\ell m}(\theta) \hat{\mathbf{r}}+\frac{f_{\ell}\left(x_{l}\right)}{x_{l}}\left[Y_{\ell m}^{\prime}(\theta) \hat{\boldsymbol{\theta}}+\chi_{\ell m}(\theta) \hat{\boldsymbol{\phi}}\right], \quad \text { for } \ell \geqslant 0, \\
\mathbf{F}_{1 \ell m}(r, \theta)=\frac{f_{\ell}\left(x_{t}\right)}{\psi_{\ell}}\left[\chi_{\ell m}(\theta) \hat{\boldsymbol{\theta}}+Y_{\ell m}^{\prime}(\theta) \hat{\boldsymbol{\phi}}\right], \quad \text { for } \ell \geqslant 1, \\
\mathbf{F}_{2 \ell m}(r, \theta)=\frac{-1}{\psi_{\ell} x_{t}}\left\{\psi_{\ell}^{2} f_{\ell}\left(x_{t}\right) Y_{\ell m}(\theta) \hat{\mathbf{r}}+\left[x_{t} f_{\ell}^{\prime}\left(x_{t}\right)+f_{\ell}\left(x_{t}\right)\right]\left[Y_{\ell m}^{\prime}(\theta) \hat{\boldsymbol{\theta}}+\chi_{\ell m}(\theta) \hat{\boldsymbol{\phi}}\right]\right\}, \text { for } \ell \geqslant 1,
\end{gathered}
$$

with $f_{\ell}$ denoting $j_{\ell}$ or $h_{\ell}^{+}, \psi_{\ell}=\sqrt{\ell(\ell+1)}, Y_{\ell m}(\theta) \equiv Y_{\ell m}(\theta, \phi=0), x_{v}=q_{v} r$, and

$$
\begin{gathered}
Y_{\ell m}^{\prime}(\theta) \equiv \frac{d Y_{\ell m}(\theta)}{d \theta}=\alpha_{\ell}^{m} Y_{\ell m+1}(\theta)-\alpha_{\ell}^{-m} Y_{\ell m-1}(\theta), \\
\chi_{\ell m}(\theta) \equiv \psi_{\ell} X_{\ell m ; \theta}(\theta, \phi=0)=\alpha_{\ell}^{-m} \cos \theta Y_{\ell m-1}(\theta)-m \sin \theta Y_{\ell m}(\theta)+\alpha_{\ell}^{m} \cos \theta Y_{\ell m+1}(\theta), \\
\chi_{\ell m}^{\prime}(\theta) \equiv \frac{d \chi_{\ell m}(\theta)}{d \theta}=\alpha_{\ell}^{-m} \cos \theta Y_{\ell m-1}^{\prime}(\theta)-m \sin \theta Y_{\ell m}^{\prime}(\theta)+\alpha_{\ell}^{m} \cos \theta Y_{\ell m+1}^{\prime}(\theta)-\alpha_{\ell}^{-m} \sin \theta Y_{\ell m-1}(\theta) \\
-m \cos \theta Y_{\ell m}(\theta)-\alpha_{\ell}^{m} \sin \theta Y_{\ell m+1}(\theta),
\end{gathered}
$$

where $\alpha_{\ell}^{m}=\frac{1}{2}[(\ell-m)(\ell+m+1)]^{1 / 2}$. 
Correspondingly, the surface traction associated with $\mathbf{F}_{\mathrm{L}}$ or $\overline{\mathbf{F}}_{\mathrm{L}}$ has also the form $\mathbf{t}\left[\mathbf{F}_{\mathrm{L}}(r, \theta, \phi)\right]=\left[t_{\mathbf{F L} ; 1}(r, \theta) \hat{\mathbf{r}}+t_{\mathbf{F L} ; 2}(r, \theta) \hat{\boldsymbol{\theta}}-\right.$ $\left.\mathrm{i}_{\mathbf{F L} ; 3}(r, \theta) \hat{\boldsymbol{\phi}}\right] \exp (\mathrm{i} m \phi)$ or $\mathbf{t}\left[\overline{\mathbf{F}}_{\mathrm{L}}(r, \theta, \phi)\right]=\left[t_{\mathbf{F L} ; 1}(r, \theta) \hat{\mathbf{r}}+t_{\mathbf{F L} ; 2}(r, \theta) \hat{\boldsymbol{\theta}}+\mathrm{i} t_{\mathbf{F L} ; 3}(r, \theta) \hat{\boldsymbol{\phi}}\right] \exp (-\mathrm{i} m \phi)$, respectively. Therefore,

$$
\mathbf{F}_{\mathrm{L}}^{(1)}(r, \theta, \phi) \cdot \mathbf{t}\left[\overline{\mathbf{F}}_{\mathrm{L}^{\prime}}^{(2)}(r, \theta, \phi)\right]=\mathbf{F}_{\mathrm{L}}^{(1)}(r, \theta) \cdot \mathbf{t}_{\mathbf{F}^{(2)} \mathbf{L}^{\prime}}(r, \theta) \exp \left[\mathrm{i}\left(m-m^{\prime}\right) \phi\right],
$$

and

$$
\mathbf{t}\left[\mathbf{F}_{\mathrm{L}}^{(1)}(r, \theta, \phi)\right] \cdot \overline{\mathbf{F}}_{\mathrm{L}^{\prime}}^{(2)}(r, \theta, \phi)=\mathbf{t}_{\mathbf{F}^{(1)} \mathrm{L}}(r, \theta) \cdot \mathbf{F}_{\mathrm{L}^{\prime}}^{(2)}(r, \theta) \exp \left[\mathrm{i}\left(m-m^{\prime}\right) \phi\right]
$$

where

$$
\begin{aligned}
& \frac{n r}{\rho c_{l}^{2}} \mathbf{t}_{\mathbf{F} 0 \ell m}(r, \theta)=\left(2 \frac{c_{t}^{2}}{c_{l}^{2}}-1\right) x_{l} f_{\ell}\left(x_{l}\right) Y_{\ell m}(\theta) \mathbf{n}+\frac{2}{x_{l}} \frac{c_{t}^{2}}{c_{l}^{2}}\left(\left\{\left[\left(\psi_{\ell}^{2}-x_{l}^{2}\right) f_{\ell}\left(x_{l}\right)-2 x_{l} f_{\ell}^{\prime}\left(x_{l}\right)\right]\right.\right. \\
& \left.\times Y_{\ell m}(\theta) \hat{\mathbf{r}}+\left[x_{l} f_{\ell}^{\prime}\left(x_{l}\right)-f_{\ell}\left(x_{l}\right)\right]\left[Y_{\ell m}^{\prime}(\theta) \hat{\boldsymbol{\theta}}+\chi_{\ell m}(\theta) \hat{\boldsymbol{\phi}}\right]\right\}-\frac{r^{\prime}(\theta)}{r(\theta)}\left\{\left[x_{l} f_{\ell}^{\prime}\left(x_{l}\right)-f_{\ell}\left(x_{l}\right)\right] Y_{\ell m}^{\prime}(\theta) \hat{\mathbf{r}}\right. \\
& \left.\left.+\left[x_{l} f_{\ell}^{\prime}\left(x_{l}\right) Y_{\ell m}(\theta)+f_{\ell}\left(x_{l}\right) Y_{\ell m}^{\prime \prime}(\theta)\right] \hat{\boldsymbol{\theta}}+f_{\ell}\left(x_{l}\right) \chi_{\ell m}^{\prime}(\theta) \hat{\boldsymbol{\phi}}\right\}\right), \\
& \frac{n r}{\rho c_{t}^{2}} \mathbf{t}_{\mathbf{F} 1 \ell m}(r, \theta)=\frac{2}{\psi_{\ell}}\left\{x_{t} f_{\ell}^{\prime}\left(x_{t}\right)\left[\chi_{\ell m}(\theta) \hat{\boldsymbol{\theta}}+Y_{\ell m}^{\prime}(\theta) \hat{\boldsymbol{\phi}}\right]-\frac{r^{\prime}(\theta)}{r(\theta)} f_{\ell}\left(x_{t}\right)\left[-\chi_{\ell m}(\theta) \hat{\mathbf{r}}+\chi_{\ell m}^{\prime}(\theta) \hat{\boldsymbol{\theta}}+Y_{\ell m}^{\prime \prime}(\theta) \hat{\boldsymbol{\phi}}\right]\right\} \\
& -\frac{1}{\psi_{\ell}}\left(\left[x_{t} f_{\ell}^{\prime}\left(x_{t}\right)+f_{\ell}\left(x_{t}\right)\right]\left\{\chi_{\ell m}(\theta)\left[\frac{r^{\prime}(\theta)}{r(\theta)} \hat{\mathbf{r}}+\hat{\boldsymbol{\theta}}\right]+Y_{\ell m}^{\prime}(\theta) \hat{\boldsymbol{\phi}}\right\}+\frac{r^{\prime}(\theta)}{r(\theta)} f_{\ell}\left(x_{t}\right) \psi_{\ell}^{2} Y_{\ell m}(\theta) \hat{\boldsymbol{\phi}}\right), \\
& \frac{n r}{\rho c_{t}^{2}} \mathbf{t}_{\mathbf{F} 2 \ell m}(r, \theta)=-\frac{2}{\psi_{\ell} x_{t}}\left(\psi_{\ell}^{2}\left[x_{t} f_{\ell}^{\prime}\left(x_{t}\right)-f_{\ell}\left(x_{t}\right)\right] Y_{\ell m}(\theta) \hat{\mathbf{r}}+\left[\left(\psi_{\ell}^{2}-x_{t}^{2}-1\right) f_{\ell}\left(x_{t}\right)-x_{t} f_{\ell}^{\prime}\left(x_{t}\right)\right]\left[Y_{\ell m}^{\prime}(\theta) \hat{\boldsymbol{\theta}}+\chi_{\ell m}(\theta) \hat{\boldsymbol{\phi}}\right]\right. \\
& -\frac{r^{\prime}(\theta)}{r(\theta)}\left\{\left[\left(\psi_{\ell}^{2}-1\right) f_{\ell}\left(x_{t}\right)-x_{t} f_{\ell}^{\prime}\left(x_{t}\right)\right] Y_{\ell m}^{\prime}(\theta) \hat{\mathbf{r}}+\left\{f_{\ell}\left(x_{t}\right)\left[\psi_{\ell}^{2} Y_{\ell m}(\theta)+Y_{\ell m}^{\prime \prime}(\theta)\right]+x_{t} f_{\ell}^{\prime}\left(x_{t}\right) Y_{\ell m}^{\prime \prime}(\theta)\right\} \hat{\boldsymbol{\theta}}\right. \\
& \left.\left.+\left\{\left[x_{t} f_{\ell}^{\prime}\left(x_{t}\right)+f_{\ell}\left(x_{t}\right)\right] \chi_{\ell m}^{\prime}(\theta) \hat{\boldsymbol{\phi}}\right\}\right\}\right)-\frac{x_{t} f_{\ell}\left(x_{t}\right)}{\psi_{\ell}}\left\{Y_{\ell m}^{\prime}(\theta)\left[\frac{r^{\prime}(\theta)}{r(\theta)} \hat{\mathbf{r}}+\hat{\boldsymbol{\theta}}\right]+\chi_{\ell m}(\theta) \hat{\boldsymbol{\phi}}\right\} .
\end{aligned}
$$

\section{A. Scatterer in host material of the same type}

If the scatterer is of the same type (fluid or solid) as the host material, both the displacement field and the surface traction are continuous at the surface:

$$
\begin{array}{r}
\mathbf{u}_{+}(\mathbf{r})=\mathbf{u}_{-}(\mathbf{r}), \\
\mathbf{t}_{+}(\mathbf{r})=\mathbf{t}_{-}(\mathbf{r}) .
\end{array}
$$

On the other hand, the interior surface fields can be expanded into regular spherical waves as follows ${ }^{43}$ :

$$
\begin{gathered}
\mathbf{u}_{-}(\mathbf{r})=\sum_{\mathrm{L}^{\prime}} \alpha_{\mathrm{L}^{\prime}} \mathbf{J}_{\mathrm{L}^{\prime}}^{\mathrm{s}}(\mathbf{r}), \\
\mathbf{t}_{-}(\mathbf{r})=\sum_{\mathrm{L}^{\prime}} \alpha_{\mathrm{L}^{\prime}} \mathbf{t}\left[\mathbf{J}_{\mathrm{L}^{\prime}}^{\mathrm{s}}(\mathbf{r})\right] .
\end{gathered}
$$

Therefore, substituting into Eqs. (29) and (30), we can express the coefficients of the incident and scattered fields in terms of those of the interior surface fields, as follows:

$$
a_{\mathrm{L}}^{0}=-\mathrm{i} \sum_{\mathrm{L}^{\prime}} \alpha_{\mathrm{L}^{\prime}} Q_{\mathrm{L}^{\prime} \mathrm{L}}^{0}, \quad a_{\mathrm{L}}^{+}=\mathrm{i} \sum_{\mathrm{L}^{\prime}} \alpha_{\mathrm{L}^{\prime}} Q_{\mathrm{L}^{\prime} \mathrm{L}}^{+},
$$

with

$$
Q_{P^{\prime} \ell^{\prime} m^{\prime} ; P \ell m}^{0}=2 \pi \delta_{m m^{\prime}} \int_{0}^{\pi} d \theta \sin \theta x_{v} \frac{n r}{\rho c_{v}^{2}}\left\{\mathbf{J}_{P^{\prime} \ell^{\prime} m}^{\mathrm{s}}(r, \theta) \cdot \mathbf{t}_{\mathbf{H} P \ell m}(r, \theta)-\mathbf{t}_{\mathbf{J}^{\mathrm{s}} P^{\prime} \ell^{\prime} m}(r, \theta) \cdot \mathbf{H}_{P \ell m}(r, \theta)\right\},
$$

and $Q_{\mathrm{L}^{\prime} \mathrm{L}}^{+}$given by the same expressions as $Q_{\mathrm{L}^{\prime} \mathrm{L}}^{0}$ with $\mathbf{J}$ in the place of $\mathbf{H}$. With the help of Eqs. (A18), Eq. (31) leads directly to Eq. (32).

\section{B. Fluid scatterer in solid host material}

In the case of a fluid scatterer $\left(\mu_{\mathrm{s}}=0\right)$ in a solid host material, the field inside the scatterer cannot sustain shear waves. Thus, only the normal component of the displacement field is continuous at the surface $S$ and the appropriate boundary conditions are

$$
\hat{\mathbf{n}} \cdot \mathbf{u}_{+}(\mathbf{r})=\hat{\mathbf{n}} \cdot \mathbf{u}_{-}(\mathbf{r}), \quad \hat{\mathbf{n}} \cdot \mathbf{t}_{+}(\mathbf{r})=\hat{\mathbf{n}} \cdot \mathbf{t}_{-}(\mathbf{r}), \quad \hat{\mathbf{n}} \times \mathbf{t}_{+}(\mathbf{r})=0 .
$$


The interior surface fields can be expanded into regular irrotational spherical waves ${ }^{43}$ :

$$
\begin{aligned}
& \mathbf{u}_{-}(\mathbf{r})=\sum_{\ell^{\prime} m^{\prime}} \alpha_{0 \ell^{\prime} m^{\prime}} \mathbf{J}_{0 \ell^{\prime} m^{\prime}}(\mathbf{r}), \\
& \mathbf{t}_{-}(\mathbf{r})=\sum_{\ell^{\prime} m^{\prime}} \alpha_{0 \ell^{\prime} m^{\prime}} \mathbf{t}\left[\mathbf{J}_{0 \ell^{\prime} m^{\prime}}^{\mathrm{s}}(\mathbf{r})\right] .
\end{aligned}
$$

The remaining tangential exterior surface fields can be expanded into regular divergenceless spherical waves as follows ${ }^{43}$ :

$$
\hat{\mathbf{n}} \times \mathbf{u}_{+}(\mathbf{r}) \times \hat{\mathbf{n}}=\sum_{P^{\prime}=1,2} \sum_{\ell^{\prime} m^{\prime}} \alpha_{P^{\prime} \ell^{\prime} m^{\prime}} \hat{\mathbf{n}} \times \mathbf{J}_{P^{\prime} \ell^{\prime} m^{\prime}}(\mathbf{r}) \times \hat{\mathbf{n}} .
$$

Applying these boundary conditions to Eqs. (29) and (30) and using the identity $\mathbf{u}_{+}(\mathbf{r})=\left[\hat{\mathbf{n}} \cdot \mathbf{u}_{+}(\mathbf{r})\right] \hat{\mathbf{n}}+\hat{\mathbf{n}} \times \mathbf{u}_{+}(\mathbf{r}) \times \hat{\mathbf{n}}$ we can express the expansion coefficients of the incident and scattered fields through Eqs. (A18), with

$$
\begin{gathered}
Q_{0 \ell^{\prime} m^{\prime} ; P \ell m}^{0}=2 \pi \delta_{m m^{\prime}} \int_{0}^{\pi} d \theta \sin \theta x_{v} \frac{n r}{\rho c_{v}^{2}}\left\{\hat{\mathbf{n}} \cdot \mathbf{J}_{0 \ell^{\prime} m}^{\mathbf{s}}(r, \theta) \hat{\mathbf{n}} \cdot \mathbf{t}_{\mathbf{H} P \ell m}(r, \theta)-\hat{\mathbf{n}} \cdot \mathbf{t}_{\mathbf{J}^{\mathrm{s}} 0 \ell^{\prime} m}(r, \theta) \hat{\mathbf{n}} \cdot \mathbf{H}_{P \ell m}(r, \theta)\right\}, \\
Q_{P^{\prime} \ell^{\prime} m^{\prime} ; P \ell m}^{0}=2 \pi \delta_{m m^{\prime}} \int_{0}^{\pi} d \theta \sin \theta x_{v} \frac{n r}{\rho c_{v}^{2}}\left\{\mathbf{J}_{P^{\prime} \ell^{\prime} m}(r, \theta) \cdot \mathbf{t}_{\mathbf{H} P \ell m}(r, \theta)-\hat{\mathbf{n}} \cdot \mathbf{J}_{P^{\prime} \ell^{\prime} m}(r, \theta) \hat{\mathbf{n}} \cdot \mathbf{t}_{\mathbf{H} P \ell m}(r, \theta)\right\}, \quad \text { for } \quad P^{\prime} \neq 0,
\end{gathered}
$$

and $Q_{\mathrm{L}^{\prime} \mathrm{L}}^{+}$given by the same expressions as $Q_{\mathrm{L}^{\prime} \mathrm{L}}^{0}$ with $\mathbf{J}$ in the place of $\mathbf{H}$.

\section{Solid scatterer in fluid host material}

In the case of a solid scatterer in a fluid host material $(\mu=0)$ the external fields cannot sustain shear waves and the exterior surface tractions are always normal to the surface. Thus, only the normal component of the displacement field is continuous at the surface $S$ and the appropriate boundary conditions are

$$
\hat{\mathbf{n}} \cdot \mathbf{u}_{+}(\mathbf{r})=\hat{\mathbf{n}} \cdot \mathbf{u}_{-}(\mathbf{r}), \quad \hat{\mathbf{n}} \cdot \mathbf{t}_{+}(\mathbf{r})=\hat{\mathbf{n}} \cdot \mathbf{t}_{-}(\mathbf{r}), \quad \hat{\mathbf{n}} \times \mathbf{t}_{-}(\mathbf{r})=0 .
$$

The interior surface fields can be expanded into regular spherical waves ${ }^{45}$ :

$$
\begin{gathered}
\mathbf{u}_{-}(\mathbf{r})=\sum_{L^{\prime}} \gamma_{L^{\prime}} \mathbf{J}_{L^{\prime}}(\mathbf{r}), \\
\mathbf{t}_{-}(\mathbf{r})=\sum_{L^{\prime}} \gamma_{L^{\prime}} \mathbf{t}\left[\mathbf{J}_{L^{\prime}}^{\mathrm{s}}(\mathbf{r})\right] .
\end{gathered}
$$

In the host medium, where only longitudinal waves exist, the exterior surface traction is normal to the surface of the scatterer. Therefore, applying the first of Eqs. (A26) to Eq. (23) we find that

$$
a_{\ell m}^{0}=-\mathrm{i} \sum_{P^{\prime} \ell^{\prime} m^{\prime}} \gamma_{P^{\prime} \ell^{\prime} m^{\prime}} P_{P^{\prime} \ell^{\prime} m^{\prime} ; \ell m}^{0}, \quad a_{\ell m}^{+}=\mathrm{i} \sum_{P^{\prime} \ell^{\prime} m^{\prime}} \gamma_{P^{\prime} \ell^{\prime} m^{\prime}} P_{P^{\prime} \ell^{\prime} m^{\prime} ; \ell m}^{+},
$$

with

$$
P_{P^{\prime} \ell^{\prime} m^{\prime} ; \ell m}^{0}=2 \pi \delta_{m m^{\prime}} \int_{0}^{\pi} d \theta \sin \theta x_{l} \frac{n r}{\rho c_{l}^{2}}\left\{\hat{\mathbf{n}} \cdot \mathbf{J}_{P^{\prime} \ell^{\prime} m}^{\mathrm{s}}(r, \theta) \hat{\mathbf{n}} \cdot \mathbf{t}_{\mathbf{H} 0 \ell m}(r, \theta)-\hat{\mathbf{n}} \cdot \mathbf{t}_{\mathbf{J}^{\mathrm{s}} P^{\prime} \ell^{\prime} m}(r, \theta) \hat{\mathbf{n}} \cdot \mathbf{H}_{0 \ell m}(r, \theta)\right\}
$$

and $P_{P^{\prime} \ell^{\prime} m^{\prime} ; \ell m}^{+}$given by the same expressions as $P_{P^{\prime} \ell^{\prime} m^{\prime} ; \ell m}^{0}$ with $\mathbf{J}$ in the place of $\mathbf{H}$. Of course, now, $\mathbf{P}^{0}$ and $\mathbf{P}^{+}$are not square matrices and thus cannot lead to the determination of the $T$ matrix analogously to Eq. (32). This can be achieved by exploiting, also, the third of the boundary conditions (A26). We expand the normal component of the exterior surface displacement field into irrotational regular spherical waves

$$
\hat{\mathbf{n}} \cdot \mathbf{u}_{+}(\mathbf{r})=\sum_{\ell^{\prime} m^{\prime}} \alpha_{\ell^{\prime} m^{\prime}} \hat{\mathbf{n}} \cdot \mathbf{J}_{0 \ell^{\prime} m^{\prime}}(\mathbf{r}) .
$$

From the first of Eqs. (A26) we have $\mathbf{u}_{-}(\mathbf{r})=\hat{\mathbf{n}} \hat{\mathbf{n}} \cdot \mathbf{u}_{+}(\mathbf{r})+\hat{\mathbf{n}} \times \mathbf{u}_{-}(\mathbf{r}) \times \hat{\mathbf{n}}$. Furthermore, using the relevant expansions for the exterior and interior surface fields in Eq. (25), we obtain

$$
\sum_{\ell^{\prime} m^{\prime}} \alpha_{\ell^{\prime} m^{\prime}} C_{\ell^{\prime} m^{\prime} ; P \ell m}+\sum_{P^{\prime} \ell^{\prime} m^{\prime}} \gamma_{P^{\prime} \ell^{\prime} m^{\prime}} R_{P^{\prime} \ell^{\prime} m^{\prime} ; P \ell m}=0,
$$

where

$$
C_{\ell^{\prime} m^{\prime} ; P \ell m}=2 \pi \delta_{m m^{\prime}} \int_{0}^{\pi} d \theta \sin \theta \frac{n r^{2}}{\rho_{\mathrm{s}} c_{\mathrm{s} v}^{2}} \hat{\mathbf{n}} \cdot \mathbf{J}_{0 \ell^{\prime} m}(r, \theta) \hat{\mathbf{n}} \cdot \mathbf{t}_{\mathbf{J}^{\mathrm{s}} P \ell m}(r, \theta)
$$


and

$$
R_{P^{\prime} \ell^{\prime} m^{\prime} ; P \ell m}=2 \pi \delta_{m m^{\prime}} \int_{0}^{\pi} d \theta \sin \theta \frac{n r^{2}}{\rho_{\mathrm{s}} c_{\mathrm{s} v}^{2}}\left\{\mathbf{J}_{P^{\prime} \ell^{\prime} m}^{\mathrm{s}}(r, \theta) \cdot \mathbf{t}_{\mathbf{J}^{\mathrm{s}} P \ell m}(r, \theta)-\hat{\mathbf{n}} \cdot \mathbf{J}_{P^{\prime} \ell^{\prime} m}^{\mathrm{s}}(r, \theta) \hat{\mathbf{n}} \cdot \mathbf{t}_{\mathbf{J}^{\mathrm{s}} P \ell m}(r, \theta)-\hat{\mathbf{n}} \cdot \mathbf{t}_{\mathbf{J}^{\mathrm{s}} P^{\prime} \ell^{\prime} m}(r, \theta) \hat{\mathbf{n}} \cdot \mathbf{J}_{P \ell m}^{\mathrm{s}}(r, \theta)\right\} .
$$

Equations (A30), (A32), (A33), and (A34), properly combined, lead to Eqs. (A18), with $\mathbf{Q}^{0(+)}=-\mathbf{C} \mathbf{R}^{-1} \mathbf{P}^{0(+)}$, from which we obtain Eq. (32).

\section{The rigid body}

The rigid scatterer is regarded as the limiting case of a very hard obstacle $\left(\rho_{\mathrm{s}} \rightarrow \infty, \lambda_{\mathrm{s}} \rightarrow \infty, \mu_{\mathrm{s}} \rightarrow \infty, c_{\mathrm{s} l}=0, c_{\mathrm{s} t}=0\right)$. In this case we assume that the field vanishes identically at the surface of the scatterer, that is,

$$
\mathbf{u}_{+}(\mathbf{r})=0 .
$$

The unknown surface traction can be expanded into regular spherical waves as follows ${ }^{43}$ :

$$
\mathbf{t}_{+}(\mathbf{r})=\sum_{\mathrm{L}^{\prime}} \frac{-\rho \omega^{2}}{q_{v}} \alpha_{\mathrm{L}^{\prime}} \mathbf{J}_{\mathrm{L}^{\prime}}(\mathbf{r})
$$

Then, applying the boundary condition to Eqs. (29) and (30), we can express the expansion coefficients of the incident and the scattered fields through Eqs. (A18), with

$$
Q_{P^{\prime} \ell^{\prime} m^{\prime} ; P \ell m}^{0}=2 \pi \delta_{m m^{\prime}} \frac{c_{v^{\prime}}^{3}}{c_{v}^{3}} \int_{0}^{\pi} d \theta \sin \theta n x_{v^{\prime}}^{2} \mathbf{J}_{P^{\prime} \ell^{\prime} m}(r, \theta) \cdot \mathbf{H}_{P \ell m}(r, \theta)
$$

and $Q_{\mathrm{L}^{\prime} \mathrm{L}}^{+}$given by the same expressions as $Q_{\mathrm{L}^{\prime} \mathrm{L}}^{0}$ with $\mathbf{J}$ in the place of $\mathbf{H}$.

\section{E. The void}

The void is regarded as the limiting case of a very soft scatterer $\left(\rho_{\mathrm{s}} \rightarrow 0, \lambda_{\mathrm{s}} \rightarrow 0, \mu_{\mathrm{s}} \rightarrow 0, c_{\mathrm{s} l}=0, c_{\mathrm{s} t}=0\right)$. In this case we assume the scatterer to have a stress-free surface, that is,

$$
\mathbf{t}_{+}(\mathbf{r})=0 .
$$

The still-unknown surface displacement field is expanded into regular spherical waves, ${ }^{43}$

$$
\mathbf{u}_{+}(\mathbf{r})=\sum_{\mathrm{L}^{\prime}} \alpha_{\mathrm{L}^{\prime}} \mathbf{J}_{\mathrm{L}^{\prime}}(\mathbf{r})
$$

and, applying the boundary condition to Eqs. (29) and (30), we find Eqs. (A18), with

$$
Q_{P^{\prime} \ell^{\prime} m^{\prime} ; P \ell m}^{0}=2 \pi \delta_{m m^{\prime}} \int_{0}^{\pi} d \theta \sin \theta x_{\nu} \mathbf{J}_{P^{\prime} \ell^{\prime} m}(r, \theta) \cdot \frac{n r}{\rho c_{v}^{2}} \mathbf{t}_{\mathbf{H} P \ell m}(r, \theta)
$$

and $Q_{\mathrm{L}^{\prime} \mathrm{L}}^{+}$given by the same expressions as $Q_{\mathrm{L}^{\prime} \mathrm{L}}^{0}$ with $\mathbf{J}$ in the place of $\mathbf{H}$.

${ }^{1}$ M. Sigalas, M. S. Kushwaha, E. N. Economou, M. Kafesaki, I. E. Psarobas, and W. Steurer, Z. Kristallogr. 220, 765 (2005).

${ }^{2}$ R. Martinez-Sala, J. Sancho, J. V. Sanchez, V. Gomez, J. Llinares, and F. Meseguer, Nature (London) 378, 241 (1995).

${ }^{3}$ W. Cheng, J. Wang, U. Jonas, G. Fytas, and N. Stefanou, Nat. Mater.5, 830 (2006).

${ }^{4}$ I. E. Psarobas, N. Stefanou, and A. Modinos, Phys. Rev. B 62, 278 (2000).

${ }^{5}$ Z. Liu, C. T. Chan, P. Sheng, A. L. Goertzen, and J. H. Page, Phys. Rev. B 62, 2446 (2000).

${ }^{6}$ R. Sainidou, N. Stefanou, I. Psarobas, and A. Modinos, Comput. Phys. Commun. 166, 197 (2005).
${ }^{7}$ C. Qiu, Z. Liu, J. Mei, and M. Ke, Solid State Commun. 134, 765 (2005).

${ }^{8}$ M. S. Kushwaha, P. Halevi, L. Dobrzynski, and B. Djafari-Rouhani, Phys. Rev. Lett. 71, 2022 (1993).

${ }^{9}$ M. Sigalas and E. N. Economou, Solid State Commun. 86, 141 (1993).

${ }^{10}$ Y. Tanaka and S. I. Tamura, Phys. Rev. B 58, 7958 (1998).

${ }^{11}$ T.-T. Wu, Z.-G. Huang, and S. Lin, Phys. Rev. B 69, 094301 (2004).

${ }^{12}$ M. M. Sigalas and N. García, J. Appl. Phys. 87, 3122 (2000).

${ }^{13}$ Y. Tanaka, Y. Tomoyasu, and S. I. Tamura, Phys. Rev. B 62, 7387 (2000). 
${ }^{14}$ A. Khelif, B. Aoubiza, S. Mohammadi, A. Adibi, and V. Laude, Phys. Rev. E 74, 046610 (2006).

${ }^{15}$ I. E. Psarobas, Phys. Rev. B 64, 012303 (2001).

${ }^{16}$ R. Sainidou, N. Stefanou, I. E. Psarobas, and A. Modinos, Phys. Rev. B 66, 024303 (2002).

${ }^{17}$ H. Zhao, Y. Liu, J. Wen, D. Yu, and X. Wen, J. Appl. Phys. 101, 123518 (2007).

${ }^{18}$ H. Zhao, J. Wen, D. Yu, and X. Wen, J. Appl. Phys. 107, 023519 (2010).

${ }^{19}$ I. E. Psarobas, N. Stefanou, and A. Modinos, Phys. Rev. B 62, 5536 (2000).

${ }^{20}$ R. Sainidou, N. Stefanou, and A. Modinos, Phys. Rev. Lett. 94, 205503 (2005).

${ }^{21}$ R. Sainidou, B. Djafari-Rouhani, and J. O. Vasseur, Phys. Rev. B 77, 094304 (2008).

${ }^{22}$ R. Sainidou and N. Stefanou, Phys. Rev. B 73, 184301 (2006).

${ }^{23}$ R. Sainidou, N. Stefanou, and A. Modinos, Phys. Rev. B 69, 064301 (2004).

${ }^{24}$ H. Chen, X. Luo, and H. Ma, Phys. Rev. B 75, 024306 (2007).

${ }^{25}$ H. Chen, K. H. Fung, H. Ma, and C. T. Chan, Phys. Rev. B 77, 224304 (2008).

${ }^{26}$ V. Leroy, A. Bretagne, M. Fink, H. Willaime, P. Tabeling, and A. Tourin, Appl. Phys. Lett. 95, 171904 (2009).

${ }^{27}$ S. Yang, J. H. Page, Z. Liu, M. L. Cowan, C. T. Chan, and P. Sheng, Phys. Rev. Lett. 88, 104301 (2002).

${ }^{28}$ S. Yang, J. H. Page, Z. Liu, M. L. Cowan, C. T. Chan, and P. Sheng, Phys. Rev. Lett. 93, 024301 (2004).

${ }^{29}$ Z. Liu, X. Zhang, Y. Mao, Y. Y. Zhu, Z. Yang, C. T. Chan, and P. Sheng, Science 289, 1734 (2000).

${ }^{30}$ Z. Liu, C. T. Chan, and P. Sheng, Phys. Rev. B 65, 165116 (2002).

${ }^{31}$ R. Sainidou, B. Djafari-Rouhani, Y. Pennec, and J. O. Vasseur, Phys. Rev. B 73, 024302 (2006).

${ }^{32}$ T. Still, R. Sainidou, M. Retsch, U. Jonas, P. Spahn, G. P. Hellmann, and G. Fytas, Nano Lett. 8, 3194 (2008).

${ }^{33}$ S. Mohammadi, A. A. Eftekhar, W. D. Hunt, and A. Adibi, Appl. Phys. Lett. 94, 051906 (2009).

${ }^{34}$ M. Eichenfield, J. Chan, R. M. Camacho, K. J. Vahala, and O. Painter, Nature (London) 462, 78 (2009).

${ }^{35}$ R. H. Olsson III and I. El-Kady, Meas. Sci. Technol. 20, 012002 (2009).

${ }^{36}$ A. Sato, Y. Pennec, N. Shingne, T. Thurn-Albrecht, W. Knoll, M. Steinhart, B. Djafari-Rouhani, and G. Fytas, ACS Nano 4, 3471 (2010).
${ }^{37}$ C. Giannetti, B. Revaz, F. Banfi, M. Montagnese, G. Ferrini, F. Cilento, S. Maccalli, P. Vavassori, G. Oliviero, E. Bontempi, L. E. Depero, V. Metlushko, and F. Parmigiani, Phys. Rev. B 76, 125413 (2007).

${ }^{38}$ B. Bonello, C. Charles, and F. Ganot, Appl. Phys. Lett. 90, 021909 (2007).

${ }^{39}$ S. Mohammadi, A. A. Eftekhar, A. Khelif, and A. Adibi, Opt. Express 18, 9164 (2010).

${ }^{40}$ A. Sukhovich, B. Merheb, K. Muralidharan, J. O. Vasseur, Y. Pennec, P. A. Deymier, and J. H. Page, Phys. Rev. Lett. 102, 154301 (2009).

${ }^{41}$ K. Imamura and S. Tamura, Phys. Rev. B 70, 174308 (2004).

${ }^{42}$ Y. Pennec, B. Djafari Rouhani, H. Larabi, A. Akjouj, J. N. Gillet, J. O. Vasseur, and G. Thabet, Phys. Rev. B 80, 144302 (2009).

${ }^{43}$ V. Varatharajulu and Y.-H. Pao, J. Acoust. Soc. Am. 60, 556 (1976).

${ }^{44}$ P. C. Waterman, J. Acoust. Soc. Am. 60, 567 (1976).

${ }^{45}$ A. Boström, J. Acoust. Soc. Am. 67, 390 (1980); 67, 1904 (1980).

${ }^{46}$ A. Lakhtakia, V. V. Varadan, and V. K. Varadan, J. Appl. Phys. 58, 4525 (1985).

${ }^{47}$ W. Kainz, J. Acoust. Soc. Am. 104, 907 (1998).

${ }^{48}$ C. Feuillade and M. F. Werby, J. Acoust. Soc. Am. 96, 3684 (1994).

${ }^{49}$ S. M. Ivansson, J. Acoust. Soc. Am. 124, 1974 (2008).

${ }^{50}$ S. M. Ivansson, Photonics Research Developments, edited by V. Nilsson, Chap. 7 (Nova Science, New York, 2008), pp. 225-245.

${ }^{51}$ G. Gantzounis and N. Stefanou, Phys. Rev. B 73, 035115 (2006); M. I. Mishchenko, L. D. Travis, and A. A. Lacis, Scattering, Absorption, and Emission of Light by Small Particles (Cambridge University Press, Cambridge, 2002).

${ }^{52}$ S. Sadat-Saleh, S. Benchabane, F. I. Baida, M.-P. Bernal, and V. Laude, J. Appl. Phys. 106, 074912 (2009).

${ }^{53}$ L. D. Landau and E. M. Lifshitz, Theory of Elasticity, 3rd ed. (Butterworth-Heinemann, Oxford, 1986).

${ }^{54}$ Y.-H. Pao and V. Varatharajulu, J. Acoust. Soc. Am. 59, 1361 (1976).

${ }^{55}$ H. Lamb, Proc. London Math. Soc. s1-13, 189 (1881).

${ }^{56} \mathrm{G}$. Gantzounis, N. Stefanou, and V. Yannopapas, J. Phys. Condens. Matter 17, 1791 (2005).

${ }^{57}$ G. Gantzounis, J. Phys. Chem. C 113, 21560 (2009).

${ }^{58}$ J. F. Cornwell, Group Theory in Physics, Vol. 1 (Academic Press, London, 1984)

${ }^{59}$ G. Gantzounis and N. Stefanou, Phys. Rev. B 74, 085102 (2006).

${ }^{60}$ Y. Pennec, B. D. Rouhani, E. H. E. Boudouti, C. Li, Y. E. Hassouani, J. O. Vasseur, N. Papanikolaou, S. Benchabane, V. Laude, and A. Martinez, Opt. Express 18, 14301 (2010). 